TRANSACTIONS OF THE

AMERICAN MATHEMATICAL SOCIETY

Volume 359, Number 2, February 2007, Pages 529-565

S 0002-9947(06)04268-1

Article electronically published on September 19, 2006

\title{
HYDRODYNAMIC LIMITS FOR KINETIC EQUATIONS AND THE DIFFUSIVE APPROXIMATION OF RADIATIVE TRANSPORT FOR ACOUSTIC WAVES
}

\author{
MANUEL PORTILHEIRO AND ATHANASIOS E. TZAVARAS
}

\begin{abstract}
We consider a class of kinetic equations, equipped with a single conservation law, which generate $L^{1}$-contractions. We discuss the hydrodynamic limit to a scalar conservation law and the diffusive limit to a (possibly) degenerate parabolic equation. The limits are obtained in the "dissipative" sense, equivalent to the notion of entropy solutions for conservation laws, which permits the use of the perturbed test function method and allows for simple proofs. A general compactness framework is obtained for the diffusive scaling in $L^{1}$. The radiative transport equations, satisfied by the Wigner function for random acoustic waves, present such a kinetic model that is endowed with conservation of energy. The general theory is used to validate the diffusive approximation of the radiative transport equation.
\end{abstract}

\section{INTRODUCTION}

We consider a class of kinetic models equipped with a single conservation law of the form

$$
\partial_{t} f(t, x, \xi)+a(\xi) \cdot \nabla_{x} f(t, x, \xi)=C(f(t, x, \cdot))(\xi),
$$

for $(t, x, \xi) \in \mathbb{R}^{+} \times \mathbb{R}^{d} \times \mathbb{X}$. The function $f(t, x, \xi)$ represents the density of particles at the point $x$ in $\mathbb{R}^{d}$ and time $t$ moving with velocity $\xi$. The variable $\xi$ may take continuous or discrete values; it may be a scalar or a vector parameter taking values in a bounded or unbounded set. If it takes discrete values, then (1.1) is a discrete velocity kinetic model. All these cases can be treated simultaneously, and for the part of the presentation that is common in all models, we employ the notation $\xi \in \mathbb{X}$.

The collision operator $C$ is a (possibly nonlinear) functional defined on $L^{1}(\mathbb{X})$. It encodes the detailed properties of a collision process. We will assume throughout that $C$ satisfies the properties:

$$
\begin{gathered}
C(0(\cdot))(\xi)=0 \\
\int_{\mathbb{X}} C(f)(\xi) d \xi=0
\end{gathered}
$$

Received by the editors September 30, 2004.

2000 Mathematics Subject Classification. Primary 35L65, 78A40, 82C40.

Key words and phrases. Diffusive limit, radiative transport.

(C)2006 American Mathematical Society Reverts to public domain 28 years from publication 
for every $f$ in $L^{1}(\mathbb{X})$ and

$$
\int_{\mathbb{X}}(C(f(\cdot))(\xi)-C(\bar{f}(\cdot))(\xi)) \operatorname{sgn}(f-\bar{f})(\xi) d \xi \leqslant 0,
$$

for every $f$ and $\bar{f}$ in $L(\mathbb{X})$. Finally, the equilibria, or the Maxwellians, $f_{e q}$, defined as the solutions of $C\left(f_{e q}\right)(\xi) \equiv 0$, form a one-parameter family in terms of the mass, $u=\int f d \xi$, which we will denote by $\mathcal{M}(u, \cdot)$ or by $\mathcal{M}(u)$. We assume that it satisfies

$$
\mathcal{M}(u) \in L^{1} \cap L^{\infty}(\mathbb{X}), \frac{d \mathcal{M}}{d u}(u, \xi)>0, \text { and } \int_{\mathbb{X}} \mathcal{M}(u, \xi) d \xi=u
$$

Under hypothesis (h1), the mass $u$ satisfies the conservation law

$$
\partial_{t} u+\operatorname{div} \int_{\mathbb{X}} a(\xi) f d \xi=0,
$$

(h2) guarantees that (1.1) is an $L^{1}$-contraction, while the Maxwellians in (h3) provide the model with a class of kinetic entropies.

The aim of this article is to develop a framework for treating the hydrodynamic and diffusive limits for collisional kinetic equations that satisfy a single conservation law and generate $L^{1}$-contractions. A number of previously studied models fit into the above framework, including relaxation approximations 13, 21, kinetic BGK models [24, 4, and the discrete kinetic models in [19, 5, 26. Most existing works concern BGK-type collision operators, and our objective is to put these works in a common framework and to develop a theory for general collision operators. Additional hypotheses on $C$ are needed, especially for the diffusive limit. These are outlined in the sequel. We apply the theory to kinetic models arising in the radiative transport of random waves (e.g. 7, 22]). Such models are naturally endowed with conservation of energy, and in the case of radiative transport for acoustic waves $C$ generates an $L^{1}$-contraction. Our analysis provides a general convergence result for the diffusive limit of the radiative transport equations for acoustic waves in multidimensional space.

A novel feature of this work is the analytical method of proof, which is based on the concept of dissipative solutions of [25] and the perturbed test function method ([9], 26]), and renders the proofs particularly simple and capable of dealing with complex models. Interestingly, the usual Hilbert expansion used for identifying the limiting behavior is now carried to the test functions, and the asymptotic analysis process is particularly appealing. In the diffusive scaling, this approach can handle cases where the estimate structure is too weak to be treated with traditional techniques.

We next outline the main results: The hyperbolic scaling concerns the limiting process $\varepsilon \rightarrow 0$ for the initial value problem

$$
\begin{aligned}
\partial_{t} f^{\varepsilon}+a(\xi) \cdot \nabla_{x} f^{\varepsilon} & =\frac{1}{\varepsilon} C\left(f^{\varepsilon}\right), \\
f(0, x, \xi) & =f_{0}(x, \xi) .
\end{aligned}
$$

(For ease of notation, here and in what follows, when $f$ is a function of $(t, x, \xi)$ we use $C(f)$ to denote the mapping $(t, x, \xi) \mapsto C(f(t, x, \cdot))(\xi)$.) To carry out this limit we employ (h0)-(h3) and place the additional assumption that

$$
\lim _{\varepsilon \rightarrow 0}\left|f^{\varepsilon}(t, x, \xi)-\mathcal{M}\left(u^{\varepsilon}(t, x), \xi\right)\right|=0 \quad \text { a.e. in } \mathbb{R}^{+} \times \mathbb{R}^{d} \times \mathbb{X} \text {. }
$$


Hypothesis (@1) follows in applications of our theory from an estimate analogous to the $\mathrm{H}$-theorem, and is justified in sections 5 and 6 for various specific models. Under hypotheses (h0)-(h3) and (f1), problem (1.2) is equipped with kinetic entropies, and the total mass $u^{\varepsilon}$ converges to the entropy solution (or equivalently to the dissipative solution) of the scalar conservation law

$$
\partial_{t} u+\operatorname{div} \int_{\mathbb{X}} a(\xi) \mathcal{M}(u, \xi) d \xi=0 .
$$

We refer to [24, 13, 21, 14, 4, 28, for convergence of various continuous or discrete BGK-type models and to 27 for the present setting of contractive collisional operators. In section 3, we give a simplified convergence proof using the setting of dissipative solutions [25], 26]. Contractive kinetic equations provide a general framework for the extension of Kruzhkov theory to kinetic models. However, not every kinetic model with one conservation law generates an $L^{1}$-contraction; see 11 . for convergence results outside the Kruzhkov setting.

Next, we consider the diffusive scaling for (1.1),

$$
\begin{aligned}
\partial_{t} f^{\varepsilon}+\frac{1}{\varepsilon} a(\xi) \cdot \nabla_{x} f^{\varepsilon} & =\frac{1}{\varepsilon^{2}} C\left(f^{\varepsilon}\right), \\
f(0, x, \xi) & =f_{0}(x, \xi) .
\end{aligned}
$$

This equation is equipped with conservation of mass:

$$
\partial_{t} u^{\varepsilon}+\frac{1}{\varepsilon} \operatorname{div} \int a(\xi) f^{\varepsilon} d \xi=0 .
$$

In addition to (h0)-(h3), we make the following assumptions: First,

$$
\int a_{i}(\xi) \mathcal{M}(u, \xi) d \xi=0 \quad \text { for every } i \text { in }\{1, \ldots, d\} .
$$

Now the hydrodynamic limit of (1.2) is the trivial equation $u_{t}=0$, and one can consider the diffusive scaling. Second, on the structure of the linearized collision operator along Maxwellians, we assume the following:

$$
\begin{aligned}
& N\left(C^{\prime}(\mathcal{M}(u))\right)=\operatorname{span}\left\{\frac{\partial \mathcal{M}}{\partial u}\right\} \text { and } \\
& R\left(C^{\prime}(\mathcal{M}(u))\right) \supset \operatorname{span}\left\{a_{j} \frac{\partial \mathcal{M}}{\partial u}: 1 \leqslant j \leqslant d\right\} .
\end{aligned}
$$

On the basis of asymptotic analysis of the diffusive scaling (see section 4) using the Hilbert expansion, the solution of (1.5) formally converges to the (possibly degenerate) parabolic equation

$$
\partial_{t} u=\sum_{i, j=1}^{d} \partial_{x_{i}} \int_{\mathbb{R}} a_{i}(\xi)\left\langle C^{\prime}(\mathcal{M}(u))^{-1}, a_{j}(\cdot) \partial_{x_{j}} \mathcal{M}(u, \cdot)\right\rangle d \xi .
$$

Indeed, we validate the convergence in the dissipative sense under two functional assumptions, namely (f1) and the assumption that the total mass

$$
\left\{u^{\varepsilon}\right\} \text { is precompact in } L_{\text {loc }}^{1}\left(\mathbb{R}^{+} \times \mathbb{R}^{d}\right) \text {. }
$$

The functional hypotheses are then justified in various circumstances: Typically, (f1) follows from an H-estimate as in the hyperbolic scaling, and this is exhibited for various models in what follows. The justification of $(\underline{22}$ is more difficult. One has available from the contraction structure control on the modulus of continuity 
in $x$, and the goal is to use the conservation of mass (1.5) with condition (h4) and a scheme on transferring $L^{1}$-modulus of continuity in $x$ into $L^{1}$-modulus of continuity in $t$ (an idea due to Kruzhkov [15] as summarized in a technical lemma from [28]). This is done in section 5.1 for the BGK-model and in section 6.3 for the radiative transport equations. The compactness analysis for the radiative transport equations uses the Fredholm structure of the linear collision operator. In the last section, it is shown how to use a strengthened version of (f1) and the Fredholm structure of the linearized collision operator in order to prove compactness and derive ( $\underline{\mathrm{f} 2}$ ) in a general setting (see section 7 ).

Our analysis encompasses various results on diffusive limits of simpler models [16, 5, 26] in the $L^{1}$-contraction framework, and extends the diffusive limit analysis to general contractive models with one conservation law. As a technique it treats the hyperbolic and diffusive scales in a common framework, and uses in an essential way the contraction structure but with very little information from the limit equation. For complementary approaches in the diffusive regime that are designed to use information from the parabolic structure of the limit equation, see $[19,20,17$. We refer to [1, 2, 3, for early work on radiative transport, to [19, 10, 12] for treatments of the diffusive limit in one space dimension and to 23] for a treatment of the diffusive limit in the context of accretive solutions for parabolic equations for a discrete model (see also the remark following Proposition 11).

The article is organized as follows. In section 2 we discuss existence and structural properties of the kinetic model (1.1) (Theorem 11) and outline the notion of dissipative solutions for accretive operators. It is noted that dissipative, entropy and regular weak solutions are all equivalent for semilinear systems or kinetic models (Theorem 3). Dissipative and entropy solutions are also equivalent for scalar conservation laws 25] and for strongly parabolic equations, but the precise relation is not yet understood at the level of degenerate parabolic equations. The convergence in the dissipative sense of the hydrodynamic limit from (1.2) to the entropy solution of the scalar conservation law (1.3) (Theorem 4) is proved in section 3 . Then the diffusive limit (1.4) is considered in section 4, and convergence is proved to the dissipative solution of (1.6). The proof of Theorem [6 is based on perturbation of test functions and an analysis of the structure of the linearized collision operator along Maxwellians. In section [5 the analysis is applied to a variety of kinetic and discrete kinetic models. Section 6 concerns the most interesting application: Papanicolaou and Ryzhik 22] derive collisional kinetic models describing the radiative transport of waves in random environments. These models provide very interesting examples of collisional models equipped with only the conservation of energy. The theory applies to the diffusive approximation of radiative transport for acoustic waves (see also [19] for the study of a simplified BGK-type model in this direction). In section 7 we use the conservation of mass together with the Fredholm structure of the linearized collision operator in order to prove compactness of mass in the diffusive regime (Proposition 11).

\section{Structural Properties of the kinetic Model}

Consider the initial value problem for the kinetic mode,

$$
\begin{aligned}
\partial_{t} f(t, x, \xi)+a(\xi) \cdot \nabla_{x} f(t, x, \xi) & =C(f(t, x, \cdot), \xi), \\
f(0, x, \xi) & =f_{0}(x, \xi),
\end{aligned}
$$


equipped with a single conservation law. In this section we discuss the notion of solution in the dissipative sense and structural properties of (2.1) under hypotheses (h0)- (h3).

2.1. Preliminaries. In preparation, we review some properties of the linear equation

$$
\begin{aligned}
\partial_{t} f(t, x, \xi)+a(\xi) \cdot \nabla_{x} f(t, x, \xi) & =g(t, x, \xi), \\
f(0, x, \xi) & =f_{0}(x, \xi) .
\end{aligned}
$$

The solution of (2.2) is computed via the method of characteristics

$$
f(t, x, \xi)=f_{0}(x-a(\xi) t, \xi)+\int_{0}^{t} g(s, x-a(\xi)(t-s), \xi) d s,
$$

and it is easy to see that: (i) if $f_{0} \in L_{x, \xi}^{1}$ and $g \in L_{t, x, \xi}^{1}$, then $f \in C\left([0, T] ; L_{x, \xi}^{1}\right)$; (ii) if $f_{0} \in L_{\mathrm{loc}, x, \xi}^{1}$ and $g \in L^{1}\left((0, T) ; L_{\mathrm{loc}, x, \xi}^{1}\right)$, then $f \in C\left([0, T] ; L_{\mathrm{loc}, x, \xi}^{1}\right)$. The solution of (2.2) can be understood in the usual mild sense, or alternatively one may define weak solutions by requiring that $f$ satisfies

$$
\begin{aligned}
- & \int_{0}^{T} \iint f\left(\varphi_{t}+a(\xi) \cdot \nabla_{x} \varphi\right) d x d \xi d t-\iint f_{0}(x, \xi) \varphi(0, x, \xi) d x d \xi \\
= & \int_{0}^{T} \iint g(t, x, \xi) \varphi(t, x, \xi) d x d \xi d t
\end{aligned}
$$

for any test function $\varphi \in C_{c}^{1}\left([0, T) \times \mathbb{R}^{d} \times \mathbb{X}\right)$.

For solutions $f$ of class $C\left([0, T] ; L_{\mathrm{loc}}^{1}\left(\mathbb{R}^{d} \times \mathbb{X}\right)\right)$ the two notions of solution coincide. Indeed, if $f$ is a weak solution, using the change of test function

$$
\psi(t, y, \xi)=\varphi(t, y+a(\xi) t, \xi),
$$

(2.4) is expressed in the equivalent form

$$
\begin{gathered}
-\int_{0}^{T} \iint f(t, y+a(\xi) t, \xi) \psi_{t} d y d \xi d t-\iint f_{0}(y, \xi) \psi(0, y, \xi) d y d \xi \\
=\int_{0}^{T} \iint g(s, y+a(\xi) s, \xi) \psi d y d \xi d s
\end{gathered}
$$

for $\psi \in C_{c}^{1}\left([0, T) \times \mathbb{R}^{d} \times \mathbb{X}\right)$. From (2.5) one obtains for $\phi \in C_{c}^{1}\left(\mathbb{R}^{d} \times \mathbb{X}\right)$

$$
\iint\left\{f(t, y+a(\xi) t, \xi)-f_{0}(y, \xi)-\int_{0}^{t} g(s, y+a(\xi) s, \xi) d s\right\} \phi(y, \xi) d y d \xi=0,
$$

which in turn implies (2.3). The converse, that a mild solution is also a weak solution, is clear.

For $K$ a compact subset of $\mathbb{R}^{d} \times \mathbb{X}$, we have the stability estimate

$$
\int_{K}|f(t, x, \xi)| d x d \xi \leqslant \int_{S_{t}(K)}\left|f_{0}\right| d x d \xi+\int_{0}^{t} \int_{S_{t-\tau}(K)}|g(\tau, x, \xi)| d \tau d x d \xi
$$

where $S_{t}(K)=\{(y, \xi): y=x-a(\xi) t,(x, \xi) \in K\}$. It implies in particular uniqueness of mild (or weak) solutions. 
2.2. Existence and structural properties of the kinetic model. Consider now the initial value problem (2.1). As was noted in the previous section, the solution $f$ of (2.1) can be understood in the weak or in the mild sense, and for solutions of class $C\left([0, T] ; L^{1}\left(\mathbb{R}^{d} \times \mathbb{X}\right)\right)$ weak and mild solutions coincide. We restrict our attention to this class, and express (2.1) in the form of an integral equation

$$
f(t, x, \xi)=f_{0}(x-a(\xi) t, \xi)+\int_{0}^{t} C(f)(\tau, x-a(\xi)(t-\tau), \xi) d \tau
$$

It is assumed that the collision operator satisfies for any given $T>0$ the bounds

$$
\begin{aligned}
\sup _{s \in[0, T]} & \|C(f)-C(\bar{f})\|_{L^{1}\left(\mathbb{R}^{d} \times \mathbb{X}\right)} \leqslant K_{1, T} \sup _{s \in[0, T]}\|f-\bar{f}\|_{L^{1}\left(\mathbb{R}^{d} \times \mathbb{X}\right)}, \\
& \quad \operatorname{ess} \sup _{s \in[0, T]}\|C(f)\|_{L^{\infty}\left(\mathbb{R}^{d} \times \mathbb{X}\right)} \leqslant K_{\infty, T}
\end{aligned}
$$

for some constants $K_{1, T}, K_{\infty, T}$ depending only on $T$ and the $L^{\infty}$ norms of $f$ and $\bar{f},\|f\|_{\infty}=\|f\|_{L^{\infty}\left((0, T) \times \mathbb{R}^{d} \times \mathbb{X}\right)}$. These hypotheses are needed for the existence part and are fulfilled for various models considered in the sequel. Henceforth, we operate under (h0)- (h3) and the assumption that $a(\xi)$ is uniformly bounded by some $M>0$,

$$
|a(\xi)| \leqslant M, \quad \text { for every } \xi \text { in } \mathbb{X} .
$$

The models (1.1), (1.2), or (1.4) satisfy conservation of mass

$$
\partial_{t} u+c(\varepsilon) \operatorname{div}_{x} \int_{\mathbb{X}} a(\xi) f d \xi=0,
$$

where $c(\varepsilon)=1$ for (1.1) and (1.2) and $c(\varepsilon)=\frac{1}{\varepsilon}$ for (1.4). Moreover, the collisional kinetic model is endowed with kinetic entropy functions associated to the Maxwellians (see [27, 28]). It is notable that all weak solutions satisfy the kinetic entropy inequalities (2.10).

Theorem 1. Let (a1), (a2) hold and $f_{0} \in L^{1} \cap L^{\infty}\left(\mathbb{R}^{d} \times \mathbb{X}\right)$. There exists a local weak solution $f \in C\left([0, T), L^{1}\left(\mathbb{R}^{d} \times \mathbb{X}\right)\right)$ defined on a maximal interval of existence. If $T<\infty$, then $\lim \sup _{t \rightarrow T^{-}}\|f(t)\|_{L^{\infty}\left(\mathbb{R}^{d} \times \mathbb{X}\right)} \rightarrow \infty$. Under hypotheses (h0) - (h3) and if the initial data, $f_{0}$, satisfies

$$
\mathcal{M}(a) \leqslant f_{0} \leqslant \mathcal{M}(b) \text { for some } a<b,
$$

then the solution $f$ is defined globally in time and satisfies

(i) The kinetic model is a contraction in $L^{1}\left(\mathbb{R}^{d} \times \mathbb{X}\right)$.

(ii) For $\kappa \in \mathbb{R}$,

$$
\partial_{t} \int_{\mathbb{X}}|f-\mathcal{M}(\kappa)| d \xi+c(\varepsilon) \operatorname{div}_{x} \int_{\mathbb{X}} a(\xi)|f-\mathcal{M}(\kappa)| d \xi \leqslant 0
$$

in $\mathcal{D}^{\prime}$, where $c(\varepsilon)=1$ for (1.1) and (1.2), and $c(\varepsilon)=\frac{1}{\varepsilon}$ for (1.4).

(iii) The domain $\prod_{\xi}[\mathcal{M}(a, \xi), \mathcal{M}(b, \xi)]$, with $a<b$, is positively invariant.

Proof. To prove local existence of mild solutions we consider the Banach spaces $X=C\left([0, \tau], L^{1}\left(\mathbb{R}^{d} \times \mathbb{X}\right)\right), Y=X \cap L^{\infty}\left((0, \tau) \times \mathbb{R}^{d} \times \mathbb{X}\right)(Y$ is dense in $X)$ and the 
closed set $F=\left\{f \in Y:\left\|f-f_{0}(x-a(\xi) t, \xi)\right\|_{\infty} \leqslant K\right\}$, where $K$ is some fixed positive constant. Let us define the map $S: Y \rightarrow Y$ by

$$
S(f)(t, x, \xi)=f_{0}(x-a(\xi) t, \xi)+\int_{0}^{t} C(f(s, x, \cdot))(\xi) d s .
$$

It is easy to check that, by (a1), for $\tau$ sufficiently small, $S: F \rightarrow F$ and is a contraction in $X$. The resulting fixed point $f$ belongs to $F$ and is a mild solution for (2.7). Furthermore, it can be continued in time as long as $\|f\|_{\infty}$ does not blow up. We omit the lengthy yet straightforward details.

Let $f$ and $\bar{f}$ be two solutions. By subtracting the corresponding equations, multiplying by $\operatorname{sgn}(f-\bar{f})$ and using (h2), we obtain

$$
\begin{aligned}
\partial_{t} \int_{\mathbb{X}}|f-\bar{f}| d \xi & +\operatorname{div}_{x} \int_{\mathbb{X}} a(\xi)|f-\bar{f}| d \xi \\
& =\frac{1}{\varepsilon} \int_{\mathbb{X}}(C(f)-C(\bar{f})) \operatorname{sgn}(f-\bar{f}) d \xi \leqslant 0 .
\end{aligned}
$$

This shows that any two solutions $f$ and $\bar{f}$ satisfy the $L^{1}$-contraction property:

$$
t \mapsto \int_{\mathbb{R}^{d}} \int_{\mathbb{X}}|f-\bar{f}|(t, x, \xi) d \xi d x \quad \text { is nonincreasing in } t .
$$

Since $\iint(f-\bar{f}) d x d \xi$ is a conserved quantity, we have

$$
t \mapsto \int_{\mathbb{R}^{d}} \int_{\mathbb{X}}(f-\bar{f})^{+}(t, x, \xi) d \xi d x \text { is nonincreasing in } t,
$$

and as a result

$$
\text { if } f_{0} \leqslant \bar{f}_{0} \text {, then } f \leqslant \bar{f} \text {. }
$$

A special class of solutions of (1.1) are the global Maxwellians $\mathcal{M}(\kappa, \xi)$. These may be used as comparison functions. For instance, if $f_{0} \leqslant \mathcal{M}(a)$, for some $a \in \mathbb{R}$, then $f(t, \cdot, \cdot) \leqslant \mathcal{M}(a)$. Part (iii) follows from this property. Finally, if $\bar{f}=\mathcal{M}(\kappa)$ in (2.11), then

$$
\int_{\mathbb{X}}\left(\partial_{t}+a(\xi) \cdot \nabla_{x}\right)|f-\mathcal{M}(\kappa, \xi)| d \xi=\frac{1}{\varepsilon} \int_{\mathbb{X}} C(f) \operatorname{sgn}(f-\mathcal{M}(\kappa, \xi)) d \xi \leqslant 0,
$$

which shows (2.10). Global existence is obtained from the $L^{\infty}$ bounds following from (2.9), (h3) and (iii). Since weak and mild solutions of class $C\left([0, T], L^{1}\left(\mathbb{R}^{d} \times \mathbb{X}\right)\right)$ coincide, weak solutions of (2.1) will satisfy the entropy inequalities (2.10).

2.3. Dissipative solutions for accretive equations. Next, we outline the notion of "dissipative solutions" introduced in [25] and examine the implications on defining a corresponding notion of solutions for collisional kinetic problems.

Consider an equation of the form

$$
A u=f,
$$

where $A: D(A) \rightarrow X$ is a (nonlinear) accretive operator defined on a subset, $D(A)$, of the Banach space $X$. The operator $A$ is accretive if for every $u$ and $v$ in $D(A)$

$$
0 \leqslant[u-v, A u-A v]_{+},
$$

where

$$
[f, g]_{+}:=\lim _{\lambda \rightarrow 0^{+}} \lambda^{-1}(\|f+g\|-\|f\|)
$$


is the Kato bracket for the norm of $X$. This inequality can be used to define a weak solution of (2.12) for $A$ accretive, by stating that $u$ solves (2.12) if

$$
0 \leqslant[u-\phi, f-A \phi]_{+}
$$

for every "nice" test function $\phi$ in some subset of the domain of $A$.

For $X=L^{1}(d \mu)$ the Kato bracket is given by the formula

$$
[f, g]_{+}=\int_{\{f \neq 0\}} \operatorname{sgn}(f) g d \mu+\int_{\{f=0\}}|g| d \mu .
$$

(Here $\operatorname{sgn}(x)=x /|x|$ if $x \neq 0, \operatorname{sgn}(0)=0$.) Thus, for a conservation law of the form

$$
u_{t}+\operatorname{div} F(u)=g
$$

the notion of dissipative solution is

$$
0 \leqslant \iint_{\{u \neq \phi\}} \operatorname{sgn}(u-\phi)\left(g-\phi_{t}-\operatorname{div} F(\phi)\right) d x d t+\iint_{\{u=\phi\}}\left|g-\phi_{t}-\operatorname{div} F(\phi)\right| d x d t .
$$

In fact, this operator is slightly better than accretive, and we can drop the second integral in the definition (see [25]). Accordingly, $u$ is a dissipative solution of (2.14) if $u$ satisfies

$$
0 \leqslant \iint_{\{u \neq \phi\}} \operatorname{sgn}(u-\phi)\left(g-\phi_{t}-\operatorname{div} F(\phi)\right) d x d t,
$$

for every $\phi$ smooth enough. In addition, as is proved in [25], the notion of dissipative solution is equivalent to the usual notion of Kruzhkov entropy solution, which is familiar from the theory of scalar conservation laws.

Dissipative solutions provide a particularly good framework to study relaxation limits (see [26] and the following sections) by using the perturbed test function method of Evans 9]. See 23] for an analogous notion of "accretive solution" for degenerate diffusion equations, and its relations with the entropy solution in [8] (see also the remark following Proposition 11).

2.4. Dissipative solutions for kinetic models. Hypothesis (h2) implies that the operator

$$
A f:=\partial_{t} f+a(\xi) \cdot \nabla_{x} f-C(f)
$$

is accretive in $L^{1}\left((0, T) ; L^{1}\left(\mathbb{R}^{d} \times \mathbb{X}\right)\right)$. Following [25], we define dissipative solutions for the equation

$$
\partial_{t} f+a(\xi) \cdot \nabla_{x} f-C(f)=0
$$

as follows.

Definition 2. A function $f$ in $C\left([0, T] ; L^{1}\left(\mathbb{R}^{d} \times \mathbb{X}\right)\right)$ is a dissipative solution of (1.1) if

$$
0 \leqslant \iiint \operatorname{sgn}(f-k-\phi)\left(-\phi_{t}-a(\xi) \cdot \nabla_{x} \phi+C(f)\right) d \xi d x d t
$$

for every smooth function $\phi$ in $C_{\mathrm{c}}^{1}\left(\mathbb{R}^{+} \times \mathbb{R}^{d} \times \mathbb{X}\right)$, and every $k$ in $\mathbb{R}$. 
Remarks. 1. There are two reasons to consider $C(f)$ instead of $C(\phi)$ in the above formula. The first is a technical one: In order to prove equivalence with entropic solutions (as in 25]) we have to use test functions of the form $k+\phi$, as above. For the above definition this can be done. By contrast, we do not in general have that $C(k+\phi)$ is in $L^{1}$. One could impose such a condition, for instance impose that $\int C(k+g) d \xi=\int C(g) d \xi$, for $g \in L^{1}(\mathbb{X})$. Such a condition is satisfied for example by the linear collision operator considered in section [6. But we avoid making this a general assumption.

2. A second reason is more philosophical. The main benefit of this formulation is that the derivatives fall on a test function. The last term involves no derivatives of $f$, hence we gain nothing by using the test function here, and we can regard it as a forcing term.

Furthermore, there is equivalence of entropy and dissipative solutions and a stronger dissipative property:

Theorem 3. Under hypotheses (h0)-(h3) the weak solution $f$ of (1.1) satisfies the entropy inequalities (2.10) and the dissipative property corresponding to the accretive operator $A$, i.e. for $\phi \in C_{\mathrm{c}}^{1}\left(\mathbb{R}^{+} \times \mathbb{R}^{d} \times \mathbb{X}\right)$

$$
0 \leqslant \iiint \operatorname{sgn}(f-\phi)\left(-\phi_{t}-a(\xi) \cdot \nabla_{x} \phi+C(\phi)\right) d \xi d x d t .
$$

Proof. Let $g=C(f)$. Then $f$ solves (1.1). For this equation we can use Lemmas 4.6 and 4.9 from 25 to conclude that the weak solution (which is an entropic solution due to the uniqueness) is a dissipative solution, and vice-versa. Finally, combining (2.15) and hypothesis (h2) gives (2.16).

\section{THE HYDRODYNAMIC LIMIT FOR DISSIPATIVE SOLUTIONS}

In this section we study the hydrodynamic limit of the kinetic equation

$$
\begin{aligned}
\partial_{t} f^{\varepsilon}(t, x, \xi)+a(\xi) \cdot \nabla_{x} f^{\varepsilon}(t, x, \xi) & =\frac{1}{\varepsilon} C\left(f^{\varepsilon}(t, x, \cdot), \xi\right), \\
f(0, x, \xi) & =f_{0}^{\varepsilon}(x, \xi) .
\end{aligned}
$$

The dissipative solution, $f^{\varepsilon}$, of this equation satisfies

$$
0 \leqslant \iiint \operatorname{sgn}\left(f^{\varepsilon}-\phi\right)\left(-\phi_{t}-a(\xi) \cdot \nabla_{x} \phi+\frac{1}{\varepsilon} C(\phi)\right) d \xi d x d t
$$

for every smooth function $\phi$ in $C_{\mathrm{c}}^{1}\left(\mathbb{R}^{+} \times \mathbb{R}^{d} \times \mathbb{X}\right)$. We will show that $u^{\varepsilon} \rightarrow u$ a.e. in $\mathbb{R}^{+} \times \mathbb{R}^{d}$ and that $u$ satisfies

$$
\partial_{t} u+\operatorname{div}_{x} \int_{\mathbb{X}} a(\xi) \mathcal{M}(u, \xi) d \xi=0
$$

in the dissipative sense, that is,

$$
0 \leqslant \iint \operatorname{sgn}(u-\psi)\left(-\partial_{t} \psi-\operatorname{div}_{x} \int_{\mathbb{X}} a(\xi) \mathcal{M}(\psi, \xi) d \xi\right) d x d t
$$

for every function $\psi$ in $k+C_{\mathrm{c}}^{1}\left(\mathbb{R}^{+} \times \mathbb{R}^{d}\right)$.

It is shown in [27] that along a subsequence $u^{\varepsilon} \rightarrow u$ a.e. and in $L_{\text {loc }}^{p}, 1 \leqslant p<\infty$, where $u$ is the entropy solution of the conservation law (1.3). In the following theorem we obtain the dissipative limit directly — we already know it has to be 
the same due to the equivalence of the notions of dissipative solutions and entropy solutions (25]). The interest is in the proof via the perturbed test function method.

We assume the structural hypotheses (h0)- (h3) and also that solutions of (1.2) satisfy (f1). In applications of Theorem 4 assumption (f1) has to be justified and typically follows from an entropy estimate (see section [5).

In the following $\omega$ denotes a modulus of continuity, i.e. a nonnegative, nondecreasing function satisfying $\lim _{\tau \rightarrow 0^{+}} \omega(\tau)=0$.

Theorem 4. If assumptions (h0)-(h3), (a2) and (f1) hold and the initial data $f_{0}$ satisfies

$$
\begin{gathered}
\mathcal{M}(a) \leqslant f_{0}^{\varepsilon} \leqslant \mathcal{M}(b) \quad \text { for some } a<b, \\
\sup _{\varepsilon>0} \iint\left|f_{0}^{\varepsilon}(x, \xi)\right| d x d \xi<\infty, \\
\iint\left|f_{0}^{\varepsilon}(x+h, \xi)-f_{0}^{\varepsilon}(x, \xi)\right| d x d \xi \leqslant \omega(|h|) \quad \text { for } h \in \mathbb{R}^{d},
\end{gathered}
$$

then along a subsequence (not relabeled) $u^{\varepsilon} \rightarrow u$ a.e. and in $L_{\mathrm{loc}}^{p}\left((0, T) \times \mathbb{R}^{d}\right)$ for any $p$ in $\{1, \ldots \infty\}$, where $u \in C\left([0, T] ; L^{1}\left(\mathbb{R}^{d}\right)\right) \cap L^{\infty}\left((0, T) \times \mathbb{R}^{d}\right)$ is the dissipative solution of (1.3).

Proof. 1. From the $L^{1}$-contraction property in Theorem 1 we obtain

$$
\begin{aligned}
\int \mid u^{\varepsilon}(t, x+h) & -u^{\varepsilon}(t, x)\left|d x \leqslant \iint\right| f^{\varepsilon}(t, x+h, \xi)-f^{\varepsilon}(t, x, \xi) \mid d x d \xi \\
& \leqslant \iint\left|f_{0}^{\varepsilon}(x+h, \xi)-f_{0}^{\varepsilon}(x, \xi)\right| d x d \xi \leqslant \omega(|h|) .
\end{aligned}
$$

Then we use an idea of Kruzhkov [15] together with the conservation law (2.8) in order to transfer the $L^{1}$-modulus of continuity in $x$ into information on the $L^{1}$ modulus of continuity in $t$. The relevant technical lemma is stated bellow, Lemma 5 , and is applied here with $G=0$ (we refer to [28, Lemma 9] for the proof). This yields

$$
\int\left|u^{\varepsilon}(t+k, x)-u^{\varepsilon}(t, x)\right| d x \leqslant K^{s t} \omega(k)
$$

( $K^{\text {st }}$ will denote a constant which, unless otherwise stated, is independent of the other relevant quantities in the relation it appears), and thus compactness of $u^{\varepsilon}$ in $L_{\text {loc }}^{1}\left(\mathbb{R}^{+} \times \mathbb{R}^{d}\right)$. It remains to prove that $u$ is indeed a dissipative solution of (1.3).

2. Being a dissipative solution of (1.2), $f^{\varepsilon}$ satisfies (3.1). Given a test function $\psi$ for (3.2), take $\phi=\mathcal{M}(\psi)$ as a test function in (3.1). By (h3), $C(\mathcal{M}(\psi))=0$, and (3.1) becomes

$$
0 \leqslant \iiint \operatorname{sgn}\left(f^{\varepsilon}-\phi\right)\left(-\phi_{t}-a(\xi) \cdot \nabla_{x} \phi\right) d \xi d x d t
$$

Using (f1), along a further subsequence if necessary, $f^{\varepsilon} \rightarrow \mathcal{M}(u, \xi)$ a.e. in $\mathbb{R}^{+} \times \mathbb{R}^{d} \times \mathbb{X}$. Let us assume for the moment that also

$$
\operatorname{sgn}\left(f^{\varepsilon}-\phi\right) \rightarrow \operatorname{sgn}(\mathcal{M}(u)-\phi)=\operatorname{sgn}(\mathcal{M}(u)-\mathcal{M}(\psi)) \stackrel{\text { h3 }}{=} \operatorname{sgn}(u-\psi) .
$$


From this we deduce that

$$
\begin{aligned}
0 & \leqslant \iiint \operatorname{sgn}(u-\psi)\left(-\partial_{t} \mathcal{M}(\psi, \xi)-a(\xi) \cdot \nabla_{x} \mathcal{M}(\psi, \xi)\right) d \xi d x d t \\
& =\iint \operatorname{sgn}(u-\psi)\left(-\partial_{t} \int \mathcal{M}(\psi) d \xi-\operatorname{div}_{x} \int a(\xi) \phi d \xi\right) d x d t \\
& =\iint \operatorname{sgn}(u-\psi)\left(-\partial_{t} \psi-\operatorname{div}_{x} \int a(\xi) \mathcal{M}(\psi, \xi) d \xi\right) d x d t,
\end{aligned}
$$

which is (3.2).

3. Next we justify the convergence of $\operatorname{sgn}\left(f^{\varepsilon}-\phi\right)$ to $\operatorname{sgn}(\mathcal{M}(u)-\phi)$. The argument is the same as that used in 26.

It is clear that we have convergence if $|\{\mathcal{M}(u)=\phi\}|=0$. If this is not the case, take test functions of the form $\phi_{\delta}=\phi+\delta \theta$, where $\theta \in \mathcal{D}$ is positive on the support of $\phi$ and $\delta>0$. We assert that there exists a sequence $\delta_{j} \rightarrow 0$ such that $\mid\{\mathcal{M}(u)=$ $\left.\phi_{\delta_{j}}\right\} \mid=0$ : Let $A_{\delta}=\left\{\mathcal{M}(u)=\phi_{\delta}\right\} \cap \operatorname{supp}(\phi)$. Given $\delta, \delta_{*}>0$, if $x \in A_{\delta} \cap A_{\delta_{*}}$, we must have $\mathcal{M}(u(x), \xi)=\phi(x, \xi)+\delta \theta(x, \xi)$ and $\mathcal{M}(u(x), \xi)=\phi(x, \xi)+\delta_{*} \theta(x, \xi)$ which implies $\delta=\delta_{*}$. Therefore, $\left\{A_{\delta}\right\}_{\delta>0}$ is a disjoint family. This means that there is at most a countable sub-family with positive measure. Hence we can certainly pick a sequence $\delta_{j} \rightarrow 0$ such that $\left|A_{\delta_{j}}\right|=0$. This proves the assertion.

4. Now we use the previous step to obtain (3.2) for $\phi_{j}=\phi_{\delta_{j}}$ :

$$
\begin{aligned}
0 \leqslant & \iiint \operatorname{sgn}\left(\mathcal{M}(u)-\phi_{j}\right)\left(-\partial_{t} \phi_{j}-a(\xi) \cdot \nabla_{x} \phi_{j}\right) d \xi d x d t \\
= & \iiint_{\{\mathcal{M}(u) \neq \phi\}} \operatorname{sgn}\left(\mathcal{M}(u)-\phi_{j}\right)\left(-\partial_{t}-a(\xi) \cdot \nabla\right) \phi_{j} d \xi d x d t \\
& -\iiint_{\{\mathcal{M}(u)=\phi\}}\left(-\partial_{t}-a(\xi) \cdot \nabla\right) \phi_{j} d \xi d x d t .
\end{aligned}
$$

The last step holds because $\operatorname{sgn}\left(\mathcal{M}(u)-\phi_{j}\right) \equiv-1$ on $\{\mathcal{M}(u)=\phi\}$. Now we let $\delta_{j} \rightarrow 0$. Noting that on $\{\mathcal{M}(u) \neq \phi\}, \operatorname{sgn}\left(\mathcal{M}(u)-\phi_{j}\right) \rightarrow \operatorname{sgn}(\mathcal{M}(u)-\phi)$ a.e., we conclude that

$$
\begin{aligned}
0 \leqslant & \iiint_{\{\mathcal{M}(u) \neq \phi\}} \operatorname{sgn}(\mathcal{M}(u)-\phi)\left(-\partial_{t}-a(\xi) \cdot \nabla\right) \phi d \xi d x d t \\
& -\iiint_{\{\mathcal{M}(u)=\phi\}}\left(-\partial_{t}-a(\xi) \cdot \nabla\right) \phi d \xi d x d t .
\end{aligned}
$$

5. Finally, if instead of considering $\phi_{\delta}=\phi+\delta \theta$ we consider $\phi_{\delta}:=\phi-\delta \theta$, then with a similar computation we deduce

$$
\begin{aligned}
0 \leqslant & \iiint_{\{\mathcal{M}(u) \neq \phi\}} \operatorname{sgn}(\mathcal{M}(u)-\phi)\left(-\partial_{t}-a(\xi) \cdot \nabla\right) \phi d \xi d x d t \\
& +\iiint_{\{\mathcal{M}(u)=\phi\}}\left(-\partial_{t}-a(\xi) \cdot \nabla\right) \phi d \xi d x d t .
\end{aligned}
$$

Adding the above inequalities and using the fact that $\operatorname{sgn}(\mathcal{M}(u)-\phi)=\operatorname{sgn}(u-\psi)$, we obtain (3.2). 
We now recall [28, Lemma 9], which is based on an idea of [15]. For a function $H=H(t, x)$ let

$$
\omega_{H}(t, h)=\sup _{|z| \leqslant h} \int_{\mathbb{R}^{d}}|H(t, x+z)-H(t, x)| d x
$$

be its $L^{1}$-modulus of continuity in $x$ and define

$$
M_{H}(k, h)=\int_{t}^{t+k} \sup _{|y|<h} \int_{\mathbb{R}^{d}}|H(\tau, x+y)-H(\tau, x)| d x d \tau=\int_{t}^{t+k} \omega_{H}(\tau, h) d \tau .
$$

Lemma 5 ([28]). Let $u, G$ and $H_{i}, i=1, \ldots, d$, be functions in $L^{1}\left((0, T) \times \mathbb{R}^{d}\right)$ satisfying

$$
\partial_{t} u+\operatorname{div}_{x} H=\mu \Delta G
$$

in the sense of distributions. There exists a constant $K>0$ such that for any $t, k, h>0$ (with $t+k<T$ ) we have

$$
\begin{gathered}
\int_{\mathbb{R}^{d}}|u(t+k, x)-u(t, x)| d x \leqslant K\left(\omega_{u}(h)+\frac{1}{h} M_{H}(k, h)+\frac{\mu}{h^{2}} M_{G}(k, h)\right) \\
\leqslant K \min _{h>0}\left(\omega_{u}(h)+\frac{k}{h} \sup _{t \leqslant \tau \leqslant t+k} \omega_{H}(k, h)+\frac{\mu k}{h^{2}} \sup _{t \leqslant \tau \leqslant t+k} \omega_{G}(k, h)\right) .
\end{gathered}
$$

\section{Diffusive Limits}

In this section we consider the diffusive limit of

$$
\begin{aligned}
\partial_{t} f(t, x, \xi)+\frac{1}{\varepsilon} a(\xi) \cdot \nabla_{x} f(t, x, \xi) & =\frac{1}{\varepsilon^{2}} C(f(t, x, \cdot))(\xi), \\
f(0, x, \xi) & =f_{0}(x, \xi) .
\end{aligned}
$$

This system corresponds to the long-time behavior of (1.2) in the scaling given by the transformation $(t, x) \mapsto(t / \varepsilon, x)$.

In addition to (h0)-(h3) we now also impose (h4) and (h5). These assumptions play a role in calculating the effective equation in the diffusive limit. The collision operator is assumed to be twice differentiable in the Fréchet sense, and we use the notations $\left\langle C^{\prime}(f), g\right\rangle$ for the derivative at the point $f$ along $g$, and $\left\langle C^{\prime \prime}(f),(g, h)\right\rangle$ for the action of the second derivative at the point $f$ of the pair $(g, h)$.

Let us first formally compute the diffusive limit equation. To this end consider a Hilbert expansion

$$
f^{\varepsilon}=f_{0}+\varepsilon f_{1}+\varepsilon^{2} f_{2}+\ldots
$$

for the solution of (1.4) and let

$$
u^{\varepsilon}=u_{0}+\varepsilon u_{1}+\varepsilon^{2} u_{2}+\ldots
$$

be the associated expansion of the mass. Matching in (1.4) the corresponding powers of $\varepsilon$, we obtain

$$
\begin{array}{ll}
\text { from the " } \varepsilon \text { " } " \text { terms } & f_{0}=\mathcal{M}\left(u_{0}, \xi\right), \\
\text { from the " } \varepsilon \text { " } " \text { terms } & a \cdot \nabla f_{0}=\left\langle C^{\prime}\left(f_{0}\right), f_{1}\right\rangle .
\end{array}
$$

The leading contribution of the conservation of mass comes, by (hㅐ), from the " $\varepsilon^{0}$ " terms and is

$$
\partial_{t} u_{0}+\operatorname{div}_{x} \int a(\xi) f_{1} d \xi=0
$$


To proceed, we need to solve (4.2) for $f_{1}$ when $f_{0}$ is the Maxwellian $\mathcal{M}\left(u_{0}\right)$. By (h5), this equation has a solution, and a general solution can be expressed as

$$
f_{1}=\left\langle C^{\prime}\left(f_{0}\right)^{-1}, a \cdot \nabla_{x} f_{0}\right\rangle+\theta=\Phi_{1}+\theta,
$$

where $\Phi_{1}$ is an inverse of $a \cdot \nabla_{x} f_{0}$ and $\theta \in N\left(C^{\prime}\left(f_{0}\right)\right)$. In general $C^{\prime}\left(f_{0}\right)$ is noninvertible, but this does not cause problems. There is a canonical choice for $\Phi_{1}$ which determines a complementary space for $N\left(C^{\prime}(\mathcal{M}(u))\right)$. Moreover, the nontrivial null space does not influence the limit equation. Indeed, $u_{0}$ satisfies the equation

$$
\partial_{t} u_{0}+\operatorname{div}_{x} \int a(\xi)\left(\Phi_{1}+\theta\right) d \xi=0
$$

where $\Phi_{1}=\left\langle C^{\prime}\left(f_{0}\right)^{-1}, a \cdot \nabla_{x} f_{0}\right\rangle$. The term $\theta \in N\left(C^{\prime}\left(f_{0}\right)\right)$ drops out from the limit

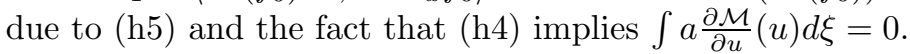

The limit equation in the diffusive regime thus becomes

$$
\partial_{t} u-\sum_{i, j=1}^{d} \partial_{x_{i}} \int_{\mathbb{X}} a_{i}(\xi)\left\langle C^{\prime}(\mathcal{M}(u))^{-1}, a_{j} \partial_{x_{j}} \mathcal{M}(u)\right\rangle d \xi=0 .
$$

Note that $u$ satisfies (4.4) in the dissipative sense if

$$
\begin{aligned}
0 \leqslant \iint & \operatorname{sgn}(u-\psi)\left(-\partial_{t} \psi\right. \\
& \left.+\sum_{i, j=1}^{d} \partial_{x_{i}} \int_{\mathbb{X}} a_{i}(\xi)\left\langle C^{\prime}(\mathcal{M}(\psi))^{-1}, a_{j} \partial_{x_{j}} \mathcal{M}(\psi)\right\rangle d \xi\right) d x d t,
\end{aligned}
$$

for any smooth $\psi$.

Next we consider a family of solutions $f^{\varepsilon}$ and the associated mass $u^{\varepsilon}$. Obtaining compactness for $\left\{u^{\varepsilon}\right\}$ is a complex issue and will be carried out for various examples in forthcoming sections. For the moment we assume (f11) and (f2 1 and carry out the convergence part. Later on compactness will be justified by taking advantage of the cancellation properties of the collision term, namely (h4). This is done for the BGK-model in Proposition 7, for the radiative transfer example of section 6 in Lemma 10 and in a general setting, with extra assumptions on the collision term, in section 7 .

Theorem 6. Assume $u^{\varepsilon} \rightarrow u$ and $f^{\varepsilon} \rightarrow \mathcal{M}(u, \xi)$ a.e. Then $u$ is a dissipative solution of (4.4), that is, it satisfies (4.5).

Proof. 1. We need to show that for any admissible test function $\psi$, (4.5) holds. Let us fix such $\psi$. Take a test function $\phi^{\varepsilon}$ for the definition of dissipative solution of (1.4) of the form $\phi^{\varepsilon}=\phi_{0}+\varepsilon \phi_{1}$. Using (h2) again we have

$$
0 \leqslant \iiint \operatorname{sgn}\left(f^{\varepsilon}-\phi^{\varepsilon}\right)\left(-\partial_{t} \phi^{\varepsilon}-\frac{1}{\varepsilon} a \cdot \nabla_{x} \phi^{\varepsilon}+\frac{1}{\varepsilon^{2}} C\left(\phi^{\varepsilon}\right)\right) d \xi d x d t .
$$

2. We want to expand $\phi^{\varepsilon}$ and gather the appropriate terms in terms of their powers of $\varepsilon$. Expanding the collision term with the formula

$$
C\left(f_{0}+f_{1}\right)=C\left(f_{0}\right)+\left\langle C^{\prime}\left(f_{0}\right), f_{1}\right\rangle+\left\langle\int_{0}^{1} \int_{0}^{t} C^{\prime \prime}\left(f_{0}+s f_{1}\right) d s d t,\left(f_{1}, f_{1}\right)\right\rangle
$$


we obtain

$$
C\left(\phi^{\varepsilon}\right)=C\left(\phi_{0}\right)+\varepsilon\left\langle C^{\prime}\left(\phi_{0}\right), \phi_{1}\right\rangle+\varepsilon^{2}\left\langle\int_{0}^{1} \int_{0}^{t} C^{\prime \prime}\left(\phi_{0}+s \varepsilon \phi_{1}\right) d s d t,\left(\phi_{1}, \phi_{1}\right)\right\rangle .
$$

Therefore we can write the integrand in (4.6) (apart from $\operatorname{sgn}\left(f^{\varepsilon}-\phi^{\varepsilon}\right)$ ) as

$$
\begin{aligned}
& -\varepsilon \partial_{t} \phi_{1}-\left(\partial_{t} \phi_{0}+a \cdot \nabla_{x} \phi_{1}-\left\langle\int_{0}^{1} \int_{0}^{t} C^{\prime \prime}\left(\phi_{0}+s \varepsilon \phi_{1}\right) d s d t,\left(\phi_{1}, \phi_{1}\right)\right\rangle\right) \\
& -\frac{1}{\varepsilon}\left(a \cdot \nabla_{x} \phi_{0}-\left\langle C^{\prime}\left(\phi_{0}\right), \phi_{1}\right\rangle\right)+\frac{1}{\varepsilon^{2}} C\left(\phi_{0}\right) .
\end{aligned}
$$

3. Since we want to let $\varepsilon \rightarrow 0$, to make the " $\varepsilon^{-2}$ " term vanish we select $\phi_{0}=$ $\mathcal{M}(\psi)$, where $\psi$ is the fixed test function. For the " $\varepsilon^{-1}$ " term we need to have

$$
\left\langle C^{\prime}\left(\phi_{0}\right), \phi_{1}\right\rangle=a \cdot \nabla_{x} \phi_{0}
$$

Using (h5) we can solve this equation for $\phi_{1}$ :

$$
\phi_{1}=\left\langle C^{\prime}\left(\phi_{0}\right)^{-1}, a \cdot \nabla_{x} \phi_{0}\right\rangle+\theta=: \Phi_{1}+\theta,
$$

where $\theta$ is in $N\left(C^{\prime}\left(\phi_{0}\right)\right)$, which is generated by $\frac{d \mathcal{M}}{d u}\left(\phi_{0}\right)$.

4. To take the limit as $\varepsilon \rightarrow 0$ assume for the moment that $\operatorname{sgn}\left(f^{\varepsilon}-\phi^{\varepsilon}\right) \rightarrow$ $\operatorname{sgn}\left(f-\phi_{0}\right)$. The limit of the rest of the terms in the integral is

$$
-\partial_{t} \phi_{0}-a(\xi) \cdot \nabla_{x} \phi_{1}+\frac{1}{2}\left\langle C^{\prime \prime}\left(\phi_{0}\right),\left(\phi_{1}, \phi_{1}\right)\right\rangle .
$$

Now observe that $\operatorname{sgn}\left(f-\phi_{0}\right)=\operatorname{sgn}(\mathcal{M}(u)-\mathcal{M}(\psi))=\operatorname{sgn}(u-\psi)$, and therefore we can take $\operatorname{sgn}(u-\psi)$ out of the $\xi$-integral. Hence,

$$
\begin{aligned}
0 \leqslant \iint \operatorname{sgn}(u-\psi)\left(-\partial_{t}\right. & \int \phi_{0} d \xi-\operatorname{div}_{x} \int a \phi_{1} d \xi \\
& \left.+\frac{1}{2} \int\left\langle C^{\prime \prime}\left(\phi_{0}\right),\left(\phi_{1}, \phi_{1}\right)\right\rangle d \xi\right) d x d t
\end{aligned}
$$

We assert that the last term vanishes. Indeed, if we take $f_{0}=\phi_{0}$ and $f_{1}=h \phi_{1}$ in (4.7) and integrate in $\xi$, then due to (h1), our choice of $\phi_{1}$ and (h4), we obtain

$$
0=h^{2}\left\langle\int_{0}^{1} \int_{0}^{t} C^{\prime \prime}\left(\phi_{0}+s h \phi_{1}\right) d s d t,\left(\phi_{1}, \phi_{1}\right)\right\rangle .
$$

Dividing by $h^{2}$ and letting $h \rightarrow 0$ we prove the assertion.

Now due to our choice of $\phi_{0}$, and (h3), we have

$$
0 \leqslant \iint \operatorname{sgn}(u-\psi)\left(-\partial_{t} \psi-\operatorname{div}_{x} \int a(\xi)\left(\Phi_{1}+\theta\right) d \xi\right) d x d t
$$

Since

$$
\int a(\xi) \theta d \xi=\frac{d}{d u} \int a(\xi) \mathcal{M}(u, \xi) d \xi=0
$$

there is no ambiguity in the above equation for any choice of an inverse for

$$
\left\langle C^{\prime}\left(\phi_{0}\right)^{-1}, a \nabla_{x} \phi_{0}\right\rangle,
$$

and we obtain (4.5). 
5. To conclude, we show the assumption $\operatorname{sgn}\left(f^{\varepsilon}-\phi^{\varepsilon}\right) \rightarrow \operatorname{sgn}\left(f-\phi_{0}\right)$ is justified. For simplicity we rewrite (4.6) as

$$
0 \leqslant \int \operatorname{sgn}\left(f^{\varepsilon}-\phi^{\varepsilon}\right) I^{\varepsilon} d z
$$

where $I^{\varepsilon}$ is smooth and converges uniformly to $I$. As in the proof of Theorem 4, we need to deal with the set $\{\mathcal{M}(u, \xi)=\phi\}$. If this set has zero measure, we can take the limit in $\varepsilon$ and obtain

$$
0 \leqslant \int \operatorname{sgn}(\mathcal{M}(u, \xi)-\phi) I d z .
$$

Because of the $\varepsilon$-perturbation to the test functions we are now using, the previous adjustment to the test function has to be done on the " $\psi$ side".

Let $\psi_{\delta}=\psi+\delta \theta$, with $\theta$ as in Theorem 4 and let $\phi_{\delta}^{\varepsilon}$ be the same as $\phi^{\varepsilon}$ above, with $\psi_{\delta}$ in place of $\psi$. Again define the sets $A_{\delta}=\left\{\mathcal{M}(u)=\phi_{\delta}\right\}$. Once more we can find a sequence $\delta_{j} \rightarrow 0$ with $\left|A_{\delta}\right|=0$. Utilizing the monotonicity of $\mathcal{M}(\cdot)$ from (h3), we deduce that

$$
0 \leqslant \int_{\{\mathcal{M}(u) \neq \phi\}} \operatorname{sgn}(\mathcal{M}(u)-\phi) I d z-\int_{\{\mathcal{M}(u)=\phi\}} I d z .
$$

If we choose $\psi_{\delta}=\psi-\delta \theta$ instead, we get the opposite sign on the last integral. Then we add the two inequalities and conclude as in step 5 of Theorem 4 .

\section{SOME KINETIC MODELS AND THEIR DIFFUSIVE LIMITS}

In this section we look at some kinetic models where the diffusive limits can be obtained from the above theory. In section 6 we deal with the most interesting application, the diffusive approximation of radiative transport for acoustic waves.

5.1. BGK-model. In the BGK-model the collision term for the transport equation has the form $\mathcal{M}(u)-f$, and the diffusive scaling for this equation becomes

$$
\partial_{t} f^{\varepsilon}(t, x, \xi)+\frac{1}{\varepsilon} a \cdot \nabla_{x} f^{\varepsilon}(t, x, \xi)=-\frac{1}{\varepsilon^{2}}\left(f^{\varepsilon}(t, x, \xi)-\mathcal{M}\left(u^{\varepsilon}(t, x), \xi\right)\right),
$$

where $u^{\varepsilon}=\int f^{\varepsilon} d \xi$. It is assumed that the Maxwellian satisfies the properties $\mathcal{M}(0) \equiv 0$ and

$$
\partial_{u} \mathcal{M}(u)>0, \quad \int_{\mathbb{X}} \mathcal{M}(u) d \xi=u, \quad \mathcal{M}(u) \in L^{1} \cap L^{\infty}(\mathbb{X}) .
$$

It is easy to check that under hat the model is contractive and satisfies all of hypotheses (h0)-(h3). In addition there is an $\mathrm{H}$-estimate for this model

$$
\begin{array}{r}
\partial_{t} \int_{\mathbb{X}} \int_{0}^{f^{\varepsilon}} \mathcal{M}^{-1}(v) d v d \xi+\operatorname{div}_{x} \frac{1}{\varepsilon} \int_{\mathbb{X}} \int_{0}^{f^{\varepsilon}} \mathcal{M}^{-1}(v) d x d \xi \\
+\frac{1}{\varepsilon^{2}} \int_{\mathbb{X}}\left(f^{\varepsilon}-\mathcal{M}\left(u^{\varepsilon}\right)\right)\left(\mathcal{M}^{-1}\left(f^{\varepsilon}\right)-u^{\varepsilon}\right) d \xi=0
\end{array}
$$

By (h3') the last integrand is positive and vanishes only if $f=\mathcal{M}(u)$. We conclude that as $\varepsilon \rightarrow 0$, along a subsequence, $f^{\varepsilon}-\mathcal{M}\left(u^{\varepsilon}\right) \rightarrow 0$ a.e. and thus (f1) is fulfilled for the BGK-model. 
For the diffusive limit we impose the structural hypothesis

$$
\int a_{i}(\xi) \mathcal{M}(u, \xi) d \xi=0, \quad \text { for every } i \in\{1, \ldots, d\} \text {. }
$$

The collision operator for the BGK is $C(f)=-f+\mathcal{M}(u)$, and the linearized collision operator is given by

$$
\left\langle C^{\prime}(f), g\right\rangle=-g+\left(\int g d \xi\right) \partial_{u} \mathcal{M}(u)
$$

Therefore,

$$
\begin{aligned}
& N\left(C^{\prime}(f)\right)=\operatorname{span}\left\{\partial_{u} \mathcal{M}(u)\right\}, \\
& R\left(C^{\prime}(f)\right)=\left\{h: \int h d \xi=0\right\},
\end{aligned}
$$

and we see that (h4) implies (h5) is fulfilled. The limiting equation in the diffusive limit becomes

$$
\partial_{t} u-\sum_{i, j=1}^{d} \partial_{x_{i}} \partial_{x_{j}} \int_{\mathbb{R}} a_{i}(\xi) a_{j}(\xi) \mathcal{M}(u, \xi) d \xi=0 .
$$

In the next theorem we validate the diffusive limit. This result has been proved in [5], and our interest here is to show an alternative argument for proving compactness that will be used later in connection to more general collision operators. Also, the limit here is understood in the dissipative sense.

Proposition 7. For the BGK model, under hypotheses (h3) and (는) if $|a(\xi)| \leqslant$ $M$ and the initial data satisfy the uniform bounds

$$
\begin{aligned}
\mathcal{M}(a) \leqslant f_{0}^{\varepsilon} \leqslant \mathcal{M}(b) \quad \text { for some } a<b, & \\
\sup _{\varepsilon>0}\left\|f_{0}^{\varepsilon}\right\|_{L^{1}}+\left\|D_{x} f_{0}^{\varepsilon}\right\|_{L^{1}}<\infty, &
\end{aligned}
$$

then $u^{\varepsilon} \rightarrow u$ a.e. and in $L_{\mathrm{loc}}^{p}\left([0, T] \times \mathbb{R}^{d}\right)$, for $1 \leqslant p<\infty$, and $u \in C\left([0, T] ; L^{1}\left(\mathbb{R}^{d}\right)\right) \cap$ $\left.L^{\infty}(0, T) \times \mathbb{R}^{d}\right)$ is a solution of (5.3) in the dissipative sense.

Proof. We have already seen that (f1) holds, and we will show below that (f2) holds as well. The result then follows from Theorem 6 .

To obtain compactness in this setting we modify the argument of Theorem 4. We again use [28, Lemma 9], but instead of applying it directly to the conservation of mass

$$
\partial_{t} u^{\varepsilon}+\operatorname{div}_{x} \frac{1}{\varepsilon} \int a(\xi) f^{\varepsilon} d \xi=0
$$

we employ an equation that better approximates the limiting response in the diffusive scale. To this end, we multiply (5.1) by $\varepsilon a(\xi)$, integrate in $\xi$ and use (h4) to obtain

$$
\partial_{t} \int \varepsilon a(\xi) f^{\varepsilon} d \xi+\operatorname{div}_{x} \int a(\xi) \otimes a(\xi) f^{\varepsilon} d \xi=-\frac{1}{\varepsilon} \int a(\xi) f^{\varepsilon} d \xi .
$$

Then (5.5) gives

$$
\partial_{t}\left(u^{\varepsilon}-\varepsilon \operatorname{div}_{x} \int a(\xi) f^{\varepsilon} d \xi\right)=\partial_{x_{i}} \partial_{x_{j}} \int a_{i}(\xi) a_{j}(\xi) f^{\varepsilon} d \xi
$$


We apply to (5.6) a variant of Lemma 5. From the $L^{1}$-contraction property

$$
\begin{aligned}
& \int\left|u^{\varepsilon}(t, x+h)-u^{\varepsilon}(t, x)\right| d x \\
& \quad \leqslant \iint\left|f^{\varepsilon}(t, x+h, \xi)-f^{\varepsilon}(t, x, \xi)\right| d x d \xi \leqslant h\left\|D_{x} f_{0}^{\varepsilon}\right\|_{L^{1}}
\end{aligned}
$$

Next, we multiply (5.6) by a function $\varphi$ in $C^{2}\left(\mathbb{R}^{d}\right)$ and integrate between $t$ and $t+\tau$ to obtain

$$
\begin{aligned}
& \int\left(u^{\varepsilon}(t+\tau, x)-u^{\varepsilon}(t, x)\right) \varphi(x) d x \\
&= \varepsilon \iint a(\xi) \cdot \nabla_{x}\left(f^{\varepsilon}(t+\tau, x, \xi)-f^{\varepsilon}(t, x, \xi)\right) \varphi(x) d x d \xi \\
&+\int_{t}^{t+\tau} \iint a_{i}(\xi) a_{j}(\xi) f^{\varepsilon}(s, x, \xi) \partial_{x_{i}} \partial_{x_{j}} \varphi(x) d x d \xi d s \\
&=: J_{1}+J_{2} .
\end{aligned}
$$

The uniform BV bound for $f_{0}^{\varepsilon}$ and the $L^{1}$-contraction property imply that $f^{\varepsilon}$ is uniformly bounded in $L^{1}\left(\mathbb{X} ; B V\left(\mathbb{R}^{d}\right)\right)$. Thus we have the following estimate for $J_{1}$ :

$$
\left|J_{1}\right| \leqslant \varepsilon K^{s t}\left\|D_{x} f_{0}^{\varepsilon}\right\|_{L^{1}} \sup _{x}|\varphi(x)|
$$

The term $J_{2}$ is estimated as in Lemma 5. First, as in the proof of [28, Lemma 9] we obtain for $h>0$

$$
\begin{aligned}
\left|J_{2}\right| & =\left|\int_{t}^{t+\tau} \int G_{i j}(s, x) \partial_{x_{i}} \partial_{x_{j}} \varphi(x) d x d s\right| \\
& \leqslant M_{G_{i j}}(\tau, h)\left(\sup _{x}\left|\partial_{x_{i}} \partial_{x_{j}} \varphi(x)\right|+\frac{1}{h^{2}} \sup _{x}|\varphi(x)|\right),
\end{aligned}
$$

where

$$
\begin{aligned}
& M_{G_{i j}}(\tau, h):=\int_{t}^{t+\tau} \sup _{|y|<h} \int\left|G_{i j}(s, x+y)-G_{i j}(s, x)\right| d x d s \\
& \quad=\int_{t}^{t+\tau} \sup _{|y|<h} \int\left|\int a_{i}(\xi) a_{j}(\xi)\left(f^{\varepsilon}(s, x+y, \xi)-f^{\varepsilon}(s, x, \xi)\right) d \xi\right| d x d s \\
& \quad \leqslant M^{2} \tau h\left\|D_{x} f_{0}^{\varepsilon}\right\|_{L^{1}} .
\end{aligned}
$$

Combining the above estimates we obtain

$$
\begin{aligned}
& \left|\int\left(u^{\varepsilon}(t+\tau, x)-u^{\varepsilon}(t, x)\right) \varphi(x) d x\right| \\
& \quad \leqslant K^{s t}\left(\varepsilon \sup _{x}|\varphi(x)|+\tau h\left(\sup _{x}\left|\partial_{x_{i}} \partial_{x_{j}} \varphi(x)\right|+\frac{1}{h^{2}} \sup _{x}|\varphi(x)|\right)\right) .
\end{aligned}
$$


The rest of the argument is classical. In (5.8) we introduce as a test function

$$
\varphi_{\delta}=\rho_{\delta} \star \operatorname{sgn}\left(u^{\varepsilon}(t+\tau, \cdot)-u^{\varepsilon}(t, \cdot)\right),
$$

where $\rho_{\delta}$ is a standard mollifier. Using (5.7) we obtain

$$
\begin{aligned}
& \int\left|u^{\varepsilon}(t+\tau, x)-u^{\varepsilon}(t, x)\right| d x \leqslant 2 \omega_{u}(t, \delta)+C\left(\varepsilon+\tau h\left(\frac{1}{\delta^{2}}+\frac{1}{h^{2}}\right)\right) \\
& \left.\leqslant K^{s t}\left(\varepsilon+\delta+\tau h\left(\frac{1}{\delta^{2}}+\frac{1}{h^{2}}\right)\right)\right),
\end{aligned}
$$

and upon optimizing in $\delta$ and $h$

$$
\int\left|u^{\varepsilon}(t+\tau, x)-u^{\varepsilon}(t, x)\right| d x \leqslant K^{s t}\left(\varepsilon+\tau^{1 / 2}\right) .
$$

Using (5.7), (5.9) and proceeding as in item 7 of the proof of Proposition 11, we see that $\left\{u^{\varepsilon}\right\}$ is sequentially precompact in $C\left([0, T] ; L_{\text {loc }}^{1}\left(\mathbb{R}^{d}\right)\right)$.

5.2. Discrete velocity systems. We consider a second example describing an interaction of particles with discrete velocities.

$$
\left\{\begin{array}{l}
\partial_{t} f_{0}+\frac{1}{\varepsilon} a_{0} \cdot \nabla_{x} f_{0}=-\frac{1}{\varepsilon^{2}} \sum_{i=1}^{d}\left(h_{i}\left(f_{0}\right)-f_{i}\right) \\
\partial_{t} f_{i}+\frac{1}{\varepsilon} a_{i} \cdot \nabla_{x} f_{i}=-\frac{1}{\varepsilon^{2}}\left(f_{i}-h_{i}\left(f_{0}\right)\right), \quad i=1, \ldots, d .
\end{array}\right.
$$

The hydrodynamic limit for this model is studied in [13]. The model can be obtained as mesoscopic scaling of a stochastic particle system ([14]). The behavior in the diffusive regime resembles the BGK model, and we give a brief outline.

It is assumed that for each $i$ in $\{0, \ldots, d\}$

$$
h_{i}(0)=0, \quad \frac{\partial h_{i}}{\partial f_{0}}>0
$$

and that the initial data satisfies the uniform bounds

$$
\sup _{\varepsilon>0, i \in\{0, \ldots, d\}}\left\|f_{i}^{\varepsilon}\right\|_{B V}+\left\|f_{i}^{\varepsilon}\right\|_{L^{\infty}}<\infty .
$$

The system is equipped with conservation of the mass $u=f_{0}+\sum f_{i}$. The Maxwellians are the vectors of the form $\left(f_{0}, h_{1}\left(f_{0}\right), \ldots, h_{d}\left(f_{0}\right)\right)$, and the model is endowed with an H-theorem:

$$
\begin{aligned}
\partial_{t}\left[\frac{1}{2} f_{0}^{2}+\sum_{i} \psi_{i}\left(f_{i}\right)\right] & +\frac{1}{\varepsilon} \operatorname{div}_{x}\left[a_{0} \frac{1}{2} f_{0}^{2}+\sum_{i} a_{i} \psi_{i}\left(f_{i}\right)\right] \\
& +\frac{1}{\varepsilon^{2}} \sum_{i}\left(f_{0}-h_{i}^{-1}\left(f_{i}\right)\right)\left(h_{i}\left(f_{0}\right)-f_{i}\right)=0,
\end{aligned}
$$

where $\psi_{i}(z)=\int_{0}^{z} h_{i}^{-1}(\tau) d \tau$ are convex. The last term is positive due to (5.11) (see [13]).

To consider the diffusive scaling, we place the structural hypothesis

$$
a_{0} f_{0}+\sum_{i=1}^{d} a_{i} h_{i}\left(f_{0}\right)=0
$$


so that hydrodynamic limit is trivial $u_{t}=0$. The expected equation in the diffusive scaling is

$$
\partial_{t} b(w)-\operatorname{div}_{x} \sum_{i=1}^{d} a_{i}\left(a_{i} \cdot \nabla_{x} h_{i}(w)\right)=0
$$

where $u=b(w):=w+\sum_{i} h_{i}(w)$.

To prove convergence in the diffusive scaling, we validate assumptions (f1) and (f2) and invoke Theorem 6. The identity (5.13) implies that

$$
b\left(f_{0}^{\varepsilon}\right)-u^{\varepsilon}=\sum_{i}\left(h_{i}\left(f_{0}^{\varepsilon}\right)-f_{i}^{\varepsilon}\right) \rightarrow 0 \quad \text { for a.e. }(t, x) .
$$

Since $b$ is increasing this means $f_{0}^{\varepsilon}-b^{-1}\left(u^{\varepsilon}\right) \rightarrow 0$, and thus (f11) is satisfied. To validate $₫ 2$ we observe that the conservation of mass

$$
\partial_{t}\left(f_{0}+\sum_{i} f_{i}\right)+\frac{1}{\varepsilon} \operatorname{div}_{x}\left(a_{0} f_{0}+\sum_{i} a_{i} f_{i}\right)=0
$$

can be expressed by using (5.14) and (5.10) in the form

$$
\partial_{t}\left(u-\varepsilon \operatorname{div}_{x} \sum_{i=1}^{d} f_{i}\right)=\operatorname{div}_{x} \sum_{i=1}^{d} a_{i}\left(a_{i} \cdot \nabla_{x}\right) f_{i} .
$$

On the one hand this implies that the diffusive limit (formally) satisfies (5.15). On the other hand one can base on (5.16) an argument as in the proof of Theorem 7 to show that $\left\{u^{\varepsilon}\right\}$ is sequentially precompact in $L_{\text {loc }}^{1}\left(\mathbb{R}^{+} \times \mathbb{R}^{d}\right)$.

5.3. A continuous kinetic model for a mixture of particles. We consider a kinetic model describing a mixture of two kinds of particles, each kind converting to the other kind of particle, but not interacting with particles of the same kind. The model has certain analogies with a discrete model studied in 26.

We consider two particle densities, $f$ and $g$, where $f$ is a function of $(t, x, \xi)$ and $g$ a function of $(t, x, \zeta)$. The kinetic variables are distinct, namely $\xi \in X$ and $\zeta \in Z$, where $X \cap Z=\emptyset$. The system governing the interaction of the two types of particles is

$$
\begin{aligned}
& \partial_{t} g+\lambda(\zeta) \cdot \nabla_{x} g=C_{1}(f, g)(\zeta):=-\int(a(\xi, \zeta) A(g)-b(\xi, \zeta) B(f)) d \xi, \\
& \partial_{t} f+\mu(\xi) \cdot \nabla_{x} f=C_{2}(f, g)(\xi):=-\int(b(\xi, \zeta) B(f)-a(\xi, \zeta) A(g)) d \zeta,
\end{aligned}
$$

where $a$ and $b$ are positive functions, and $A$ and $B$ strictly increasing with $A(0)=$ $B(0)=0$. We discuss conditions on the parameters so that the structural hypotheses from the previous sections are fulfilled. 
5.3.1. Contraction property. Clearly the mass $u:=\int g d \zeta+\int f d \xi$ is conserved. Let us check the contraction property:

$$
\begin{aligned}
& \int\left(C_{1}(f, g)-C_{1}(\bar{f}, \bar{g})\right) \operatorname{sgn}(g-\bar{g}) d \zeta+\int\left(C_{2}(f, g)-C_{2}(\bar{f}, \bar{g})\right) \operatorname{sgn}(f-\bar{f}) d \xi \\
& =-\iint \operatorname{sgn}(g-\bar{g})[a A(g)-b B(f)-a A(\bar{g})+b B(\bar{f})] \\
& \quad \quad+\operatorname{sgn}(f-\bar{f})[b B(f)-a A(g)-b B(\bar{f})+a A(\bar{g})] d \xi d \zeta \\
& =\iint(\operatorname{sgn}(f-\bar{f})-\operatorname{sgn}(g-\bar{g}))[a(A(g)-A(\bar{g}))-b(B(f)-B(\bar{f}))] d \xi d \zeta \\
& \leqslant 0,
\end{aligned}
$$

since $a, b>0$ and $A$ and $B$ are monotone. This is hypothesis (h2). Hypotheses (h0) and (h1) are obvious.

5.3.2. Entropy and Maxwellians. Maxwellians for this model will come out of an analysis of kinetic entropies and an associated "H-theorem". Let $\mathcal{A}^{\prime}=A, \mathcal{B}^{\prime}=B$ and note that $\mathcal{A}, \mathcal{B}$ are convex functions. Multiplying the first equation in (5.17) by $\psi A(g)$ and the second by $\varphi B(f)$, where $\psi=\psi(\zeta)>0, \varphi=\varphi(\xi)>0$, integrating the resulting identities in $\zeta$ and $\xi$, respectively, and adding we obtain

$$
\begin{aligned}
& \partial_{t}\left(\int \psi(\zeta) \mathcal{A}(g) d \zeta+\int \varphi(\xi) \mathcal{B}(f) d \xi\right) \\
& \quad+\operatorname{div}_{x}\left(\int \lambda(\zeta) \psi(\zeta) \mathcal{A}(g) d \zeta+\int \mu(\xi) \varphi(\xi) \mathcal{B}(f) d \xi\right) \\
& \quad+\iint(\psi(\zeta) A(g)-\varphi(\xi) B(f))(a(\xi, \zeta) A(g)-b(\xi, \zeta) B(f)) d \xi d \zeta=0 .
\end{aligned}
$$

Assuming the functions $a$ and $b$ have the form

$$
\left\{\begin{array}{l}
a(\xi, \zeta)=d(\xi, \zeta) \psi(\zeta), \\
b(\xi, \zeta)=d(\xi, \zeta) \varphi(\xi),
\end{array} \quad d>0, \psi>0, \varphi>0,\right.
$$

the last term is positive and the model is equipped with the following version of H-Theorem:

$$
\begin{aligned}
& \partial_{t}\left(\int \psi(\zeta) \mathcal{A}(g) d \zeta+\int \varphi(\xi) \mathcal{B}(f) d \xi\right) \\
& \quad+\operatorname{div}_{x}\left(\int \lambda(\zeta) \psi(\zeta) \mathcal{A}(g) d \zeta+\int \mu(\xi) \varphi(\xi) \mathcal{B}(f) d \xi\right) \\
& \quad+\iint(\psi(\zeta) A(g)-\varphi(\xi) B(f))^{2} d(\xi, \zeta) d \xi d \zeta=0
\end{aligned}
$$

The Maxwellians $g_{e q}(\zeta), f_{e q}(\xi)$ satisfy

$$
\psi(\zeta) A\left(g_{e q}(\zeta)\right)=\varphi(\xi) B\left(f_{e q}(\xi)\right)=\alpha
$$

for some constant $\alpha$ and thus can be determined by

$$
g_{e q}(\zeta)=A^{-1}\left(\frac{\alpha}{\psi(\zeta)}\right), \quad f_{e q}(\xi)=B^{-1}\left(\frac{\alpha}{\varphi(\xi)}\right), \quad \alpha \in \mathbb{R} .
$$

If we want these Maxwellians to be in $L^{1}$ we need to replace technical hypotheses relating the growth of $A, B$, with the growth of $\varphi(\xi), \psi(\zeta)$. For simplicity, we will 
work out only the case when the kinetic variables take values in compact sets, $X$ and $Z$. The total mass of a Maxwellian

$$
\begin{aligned}
m(\alpha) & :=\int_{X} f_{e q} d \xi+\int_{Z} g_{e q} d \zeta \\
& =\int_{X} B^{-1}\left(\frac{\alpha}{\varphi(\xi)}\right) d \xi+\int_{Z} A^{-1}\left(\frac{\alpha}{\psi(\zeta)}\right) d \zeta
\end{aligned}
$$

is a strictly increasing function of $\alpha$. Therefore, Maxwellians may be re-parametrized in terms of their total mass and (h3) is satisfied.

5.3.3. Hydrodynamic limit. We now consider the hydrodynamic limit for (5.17), (5.18)

$$
\begin{aligned}
\partial_{t} g+\lambda \cdot \nabla_{x} g & =-\frac{1}{\varepsilon} \int_{X} d(\psi A(g)-\varphi B(f)) d \xi, \\
\partial_{t} f+\mu \cdot \nabla_{x} f & =-\frac{1}{\varepsilon} \int_{Z} d(\varphi B(f)-\psi A(g)) d \zeta
\end{aligned}
$$

and prove the convergence of the solutions to the associated conservation law.

Proposition 8. Let the initial data satisfy uniform bounds as in (3.3) and assume $a(\xi, \zeta)=d(\xi, \zeta) \psi(\zeta), b(\xi, \zeta)=d(\xi, \zeta) \varphi(\xi)$, where $\varphi, \psi$ and $d$ are positive functions, $X$ and $Z$ are compact sets and $A$ and $B$ are strictly increasing with $A(0)=B(0)=$ 0 . Then the total mass, $u^{\varepsilon}$, of the mixture model (5.22) satisfies $u^{\varepsilon} \rightarrow u$ for a.e. $(t, x)$, where $u$ is the entropy solution of a scalar conservation law

$$
\partial_{t} u+\operatorname{div}_{x} F(u)=0 .
$$

Proof. We apply the general theory of section 3 to justify this hydrodynamic limit. We need to justify (f1). We use (5.19) and proceed as in Theorem 4 to show that along a subsequence $u^{\varepsilon} \rightarrow u$ for a.e. $(t, x)$ and $\psi(\zeta) A\left(g^{\varepsilon}(\zeta)\right)-\varphi(\xi) B\left(f^{\varepsilon}(\xi)\right) \rightarrow 0$ for a.e. $(t, x)$ and $(\zeta, \xi)$. Set

$$
\begin{array}{ll}
\alpha^{\varepsilon}(t, x, \zeta)=\psi A\left(g^{\varepsilon}\right), & \bar{\alpha}^{\varepsilon}(t, x)=\frac{1}{|Z|} \int_{Z} \alpha^{\varepsilon} d \zeta, \\
\beta^{\varepsilon}(t, x, \xi)=\varphi B\left(f^{\varepsilon}\right), & \bar{\beta}^{\varepsilon}(t, x)=\frac{1}{|X|} \int_{X} \beta^{\varepsilon} d \zeta
\end{array}
$$

and note that by the monotonicity of $A$ and $B$

$$
\begin{aligned}
& \bar{\alpha}^{\varepsilon}-\bar{\beta}^{\varepsilon} \rightarrow 0, \\
& f^{\varepsilon}-B^{-1}\left(\frac{\bar{\alpha}^{\varepsilon}}{\psi}\right) \rightarrow 0, \quad g^{\varepsilon}-A^{-1}\left(\frac{\bar{\beta}^{\varepsilon}}{\varphi}\right) \rightarrow 0, \\
& u^{\varepsilon}-\int_{X} B^{-1}\left(\frac{\bar{\alpha}^{\varepsilon}}{\varphi(\xi)}\right) d \xi-\int_{Z} A^{-1}\left(\frac{\bar{\alpha}^{\varepsilon}}{\psi(\zeta)}\right) \rightarrow 0 .
\end{aligned}
$$

Since $m(\alpha)$ in (5.21) is increasing, (5.3.3) implies $\bar{\alpha}^{\varepsilon} \rightarrow \alpha=m^{-1}(u)$ for a.e. $(t, x)$. Accordingly, $f^{\varepsilon}$ and $g^{\varepsilon}$ converge strongly to the Maxwellian with parameter $\alpha(t, x)$. The limit satisfies the scalar conservation law

$$
\begin{aligned}
& \partial_{t} m(\alpha)+\operatorname{div}_{x} G(\alpha)=0 \\
& G(\alpha)=\int_{Z} \lambda A^{-1}\left(\frac{\alpha}{\psi}\right) d \zeta+\int_{X} \mu B^{-1}\left(\frac{\alpha}{\varphi}\right) d \xi
\end{aligned}
$$


Using (5.21) the latter may be expressed in the more conventional form (5.23) with $F(u)=\left(G \circ m^{-1}\right)(u)$.

5.4. Mixture model - diffusive scaling. For the nonlinear model (5.22) it is not easy to obtain compactness of $u^{\varepsilon}$ in the diffusive scaling. Instead, we consider a linear model satisfying (5.18) and $A(z)=B(z)=z$ :

$$
\begin{aligned}
& \partial_{t} g+\frac{1}{\varepsilon} \lambda \cdot \nabla_{x} g=-\frac{1}{\varepsilon^{2}} \int_{X} d(\psi g-\varphi f) d \xi, \\
& \partial_{t} f+\frac{1}{\varepsilon} \mu \cdot \nabla_{x} f=-\frac{1}{\varepsilon^{2}} \int_{Z} d(\varphi f-\psi g) d \zeta .
\end{aligned}
$$

The H-theorem now takes the form

$$
\begin{gathered}
\partial_{t} \int_{Z} \psi \frac{g^{2}}{2} d \zeta+\int_{X} \varphi \frac{f^{2}}{2} d \xi+\frac{1}{\varepsilon} \operatorname{div}_{x} \int_{Z} \lambda \psi \frac{g^{2}}{2} d \zeta+\int_{X} \mu \varphi \frac{f^{2}}{2} d \xi \\
+\frac{1}{\varepsilon^{2}} \iint_{X \times Z}(\psi g-\varphi f)^{2} d d \xi d \zeta=0,
\end{gathered}
$$

the Maxwellians are

$$
f_{\mathrm{eq}}(\xi)=\frac{\alpha}{\varphi(\xi)}, \quad g_{\mathrm{eq}}(\zeta)=\frac{\alpha}{\psi(\zeta)},
$$

and their total mass is related to $\alpha$ by the relation

$$
m(\alpha)=\int_{X} f_{e q} d \xi+\int_{Z} g_{e q} d \zeta=\alpha\left(\int_{X} \frac{1}{\varphi} d \xi+\int_{Z} \frac{1}{\psi} d \zeta\right) .
$$

The balance hypothesis (h4) for this model is

$$
\int_{Z} \lambda(\zeta) \frac{1}{\psi(\zeta)} d \zeta+\int_{X} \mu(\xi) \frac{1}{\varphi(\xi)} d \xi=0
$$

5.4.1. Collision operator. The collision operator $\mathbf{C}$ is linear and may be expressed as

$$
\mathbf{C}\left[\begin{array}{l}
g \\
f
\end{array}\right]=\left[\begin{array}{l}
\int_{X} d(\psi g-\varphi f) d \xi \\
\int_{Z} d(\varphi f-\psi g) d \zeta
\end{array}\right]=(J \mathbf{I}-\mathbf{A})\left[\begin{array}{l}
g \\
f
\end{array}\right],
$$

where $\mathbf{I}$ is the identity, $J$ is the invertible matrix

$$
J=\left[\begin{array}{cc}
\psi(\zeta) d_{1}(\zeta) & 0 \\
0 & \varphi(\xi) d_{2}(\xi)
\end{array}\right]
$$

with

$$
d_{1}(\zeta)=\int_{X} d(\xi, \zeta) \varphi(\xi) d \xi>0, \quad d_{2}(\xi)=\int_{Z} d(\xi, \zeta) \psi(\zeta) d \zeta>0
$$

and $\mathbf{A}$ is the compact operator on $\mathcal{E}=L^{1}(Z) \times L^{1}(X)$ defined by

$$
\mathbf{A}\left[\begin{array}{l}
g \\
f
\end{array}\right]=\left[\begin{array}{l}
\int_{X} d(\xi, \zeta) \varphi(\xi) f(\xi) d \xi \\
\int_{Z} d(\xi, \zeta) \psi(\zeta) g(\zeta) d \zeta
\end{array}\right]
$$

Observe that

$$
\begin{aligned}
& \mathcal{N}(\mathbf{C})=\mathcal{N}(J \mathbf{I}-\mathbf{A})=\left\{(g, f) \in \mathcal{E}: g=\frac{\alpha}{\psi}, f=\frac{\alpha}{\varphi}\right\} \\
& \mathcal{R}(\mathbf{C})=\mathcal{N}\left(J \mathbf{I}-\mathbf{A}^{\star}\right)^{\perp}=\left\{(g, f) \in \mathcal{E}: \int_{Z} g d \zeta+\int_{X} f d \xi=0\right\}
\end{aligned}
$$


and $\mathcal{R}(\mathbf{C})$ is closed. Moreover, $\mathbf{C}: \mathcal{N}(\mathbf{C})^{\perp} \rightarrow \mathcal{R}(\mathbf{C})$ is invertible and its inverse $\mathbf{K}: \mathcal{R}(\mathbf{C}) \rightarrow \mathcal{E} / \mathcal{N}(\mathbf{C})$ is a bounded linear map

$$
\mathbf{K}\left[\begin{array}{l}
g \\
f
\end{array}\right]=\left[\begin{array}{l}
K_{1}(g, f) \\
K_{2}(g, f)
\end{array}\right] \text {. }
$$

5.4.2. Diffusive limit. The balance hypothesis (h4) for this model becomes

$$
\int_{Z} \lambda(\zeta) \frac{1}{\psi(\zeta)} d \zeta+\int_{X} \mu(\xi) \frac{1}{\varphi(\xi)} d \xi=0
$$

and (h5) follows from the above analysis of the collision operator.

We proceed to obtain the limiting equation in the diffusive scaling. The mass $u^{\varepsilon}$ satisfies

$$
\partial_{t} u^{\varepsilon}+\frac{1}{\varepsilon} \operatorname{div}_{x}\left(\int_{Z} \lambda g^{\varepsilon} d \zeta+\int_{X} \mu f^{\varepsilon} d \xi\right)=0 .
$$

By inverting the collision operator $\mathbf{C}$ we obtain from (5.24)

$$
\frac{1}{\varepsilon}\left[\begin{array}{l}
g^{\varepsilon} \\
f^{\varepsilon}
\end{array}\right]=-\mathbf{K}\left[\begin{array}{l}
\varepsilon \partial_{t} g^{\varepsilon}+\lambda \cdot \nabla_{x} g^{\varepsilon} \\
\varepsilon \partial_{t} f^{\varepsilon}+\mu \cdot \nabla_{x} f^{\varepsilon}
\end{array}\right]=-\varepsilon \partial_{t} \mathbf{K}\left[\begin{array}{l}
g^{\varepsilon} \\
f^{\varepsilon}
\end{array}\right]-\partial_{x_{j}} \mathbf{K}\left[\begin{array}{l}
\lambda_{j} g^{\varepsilon} \\
\mu_{j} f^{\varepsilon}
\end{array}\right]
$$

(here we are using the summation convention). Therefore,

$$
\begin{aligned}
& \partial_{t}\left(u^{\varepsilon}-\varepsilon \partial_{x_{i}}\left(\int_{Z} \lambda_{i} K_{1}\left(g^{\varepsilon}, f^{\varepsilon}\right) d \zeta+\int_{X} \mu_{i} K_{2}\left(g^{\varepsilon}, f^{\varepsilon}\right) d \xi\right)\right) \\
& \quad=\partial_{x_{i}} \partial_{x_{j}}\left(\int_{Z} \lambda_{i} K_{1}\left(\lambda_{j} g^{\varepsilon}, \mu_{j} f^{\varepsilon}\right) d \zeta+\int_{X} \mu_{i} K_{2}\left(\lambda_{j} g^{\varepsilon}, \mu_{j} f^{\varepsilon}\right) d \xi\right) .
\end{aligned}
$$

Equation (5.28) provides an efficient approximation of the problem in the diffusive regime. Using (5.27) and proceeding as in Theorem 9 of section 6.3 we show that, for data satisfying uniform BV bounds, the total mass is precompact in $L_{\mathrm{loc}}^{1}\left(\mathbb{R}^{+} \times \mathbb{R}^{d}\right)$ and along a subsequence $u^{\varepsilon} \rightarrow u$ for a.e. $(t, x)$.

The H-estimate ensures that $\psi(\zeta) g^{\varepsilon}(\zeta)-\varphi(\xi) f^{\varepsilon}(\xi)$ converges pointwise for a.e. $(t, x)$ and a.e. $(\zeta, \xi)$. Set $\alpha^{\varepsilon}=\frac{1}{|Z|} \int \psi g^{\varepsilon}$. Then, as in the proof of Proposition 8 we have

and thus

$$
\begin{aligned}
& \int_{X}\left|f^{\varepsilon}-\frac{1}{\varphi} \alpha^{\varepsilon}\right| d \xi \rightarrow 0, \quad \int_{Z}\left|g^{\varepsilon}-\frac{1}{\psi} \alpha^{\varepsilon}\right| d \zeta \rightarrow 0, \\
& u^{\varepsilon}-\alpha^{\varepsilon}\left(\int_{X} \frac{1}{\varphi} d \xi+\int_{Z} \frac{1}{\psi} d \zeta\right) \rightarrow 0
\end{aligned}
$$

$$
\left\{\begin{array}{l}
f^{\varepsilon} \rightarrow \frac{1}{m} \frac{1}{\varphi} u, \\
g^{\varepsilon} \rightarrow \frac{1}{m} \frac{1}{\psi} u,
\end{array} \quad \text { where } m=\int_{X} \frac{1}{\varphi} d \xi+\int_{Z} \frac{1}{\psi} d \zeta .\right.
$$

We can now pass to the limit as $\varepsilon \rightarrow 0$ in (5.28) and conclude that $u$ satisfies the equation

$$
\begin{aligned}
\partial_{t} u(t, x) & =\sum_{i, j} \frac{1}{m} D_{i j} \partial_{x_{i}} \partial_{x_{j}} u(t, x) \\
\text { with } \quad D_{i j} & =\int_{Z} \lambda_{i} K_{1}\left(\frac{\lambda_{j}}{\varphi}, \frac{\mu_{j}}{\psi}\right) d \zeta+\int_{X} \mu_{i} K_{2}\left(\frac{\lambda_{j}}{\varphi}, \frac{\mu_{j}}{\psi}\right) d \xi .
\end{aligned}
$$

Unlike the radiative transfer example of the next section, the diffusion matrix $D_{i j}$ cannot be computed explicitly for the mixture model. 


\section{DifFUSION APPROXIMATION FOR WAVES IN RANDOM MEDIA}

An important class of equations that falls under the above formalism comes from rather general symmetric hyperbolic systems of the form

$$
\begin{aligned}
& A(\mathbf{x}) \frac{\partial \mathbf{u}}{\partial t}(t, \mathbf{x})+\sum_{i} D^{i} \frac{\partial \mathbf{u}}{\partial x^{i}}(t, \mathbf{x})=0, \\
& \mathbf{u}(0, \mathbf{x})=\mathbf{u}_{0}(\mathbf{x}),
\end{aligned}
$$

where $\mathbf{u}$ is a complex $N$-vector and $\mathbf{x} \in \mathbb{R}^{3}$. The matrix $A(\mathbf{x})$ is assumed to be symmetric and positive definite while the matrices $D^{i}$ are symmetric and independent of $\mathbf{x}$ and $t$. Three particular examples of models of this type are acoustic waves, electromagnetic waves and elastic waves.

If we define the Wigner distribution for the $N$-vector solutions of this system as the $N \times N$ matrix

$$
W(t, \mathbf{x}, \mathbf{k})=\frac{1}{(2 \pi)^{d}} \int e^{i \mathbf{k} \cdot \mathbf{y}} \mathbf{u}\left(t, \mathbf{x}-\frac{\mathbf{y}}{2}\right) \mathbf{u}^{*}\left(t, \mathbf{x}+\frac{\mathbf{y}}{2}\right) d \mathbf{y},
$$

where $\mathbf{u}^{*}=\overline{\mathbf{u}}^{t}$ is the conjugate transpose of $\mathbf{u}$. Then $W(t, \mathbf{x}, \mathbf{k})$ satisfies a certain transport equation. For the above-mentioned examples this equation looks like the equation for radiative transfer $([7])$.

The general reference we are following is [22, from where we take the notation used here.

We want to understand this type of equation with small random perturbations, considering instead the system

$$
A(\mathbf{x})\left\{I+\varepsilon^{1 / 2} V\left(\frac{\mathbf{x}}{\varepsilon}\right)\right\} \frac{\partial \mathbf{u}_{\varepsilon}}{\partial t}+\sum_{i} D^{i} \frac{\partial \mathbf{u}_{\varepsilon}}{\partial x^{i}}=0
$$

where $V(\mathbf{x})$ is a matrix-valued random process with zero mean, statistically homogeneous in $\mathbf{x}$. In this case it is necessary to consider the scaled Wigner distribution matrix

$$
W^{\varepsilon}(t, \mathbf{x}, \mathbf{k})=\frac{1}{(2 \pi)^{d}} \int e^{i \mathbf{k} \cdot \mathbf{y}} \mathbf{u}_{\varepsilon}\left(t, \mathbf{x}-\varepsilon \frac{\mathbf{y}}{2}\right) \mathbf{u}_{\varepsilon}^{*}\left(t, \mathbf{x}+\varepsilon \frac{\mathbf{y}}{2}\right) d \mathbf{y} .
$$

To understand the behavior of $W^{\varepsilon}$ we can formally expand it in terms of $\varepsilon$ with a new "fast" variable $\boldsymbol{\xi}=\mathbf{x} / \varepsilon$

$$
W^{\varepsilon}(t, \mathbf{x}, \mathbf{k})=W^{(0)}(t, \mathbf{x}, \mathbf{k})+\varepsilon^{1 / 2} W^{(1)}(t, \mathbf{x}, \boldsymbol{\xi}, \mathbf{k})+\varepsilon W^{(2)}(t, \mathbf{x}, \boldsymbol{\xi}, \mathbf{k})+\ldots
$$

Then the statistical average of $W^{\varepsilon},\left\langle W^{\varepsilon}\right\rangle$ should be close to $W^{(0)}$ and satisfy the radiative transfer-like transport equation

$$
\frac{\partial W}{\partial t}+\mathbf{k} \cdot \nabla_{\mathbf{x}} W=\overline{\mathcal{L}} W
$$

where

$$
\overline{\mathcal{L}} W(\mathbf{x}, \mathbf{k})=4 \pi \int \hat{R}(\mathbf{p}-\mathbf{k}) \delta\left(\mathbf{k}^{2}-\mathbf{p}^{2}\right)(W(\mathbf{x}, \mathbf{p})-W(\mathbf{x}, \mathbf{k})) d \mathbf{p} .
$$

We can expand $W^{(0)}(t, \mathbf{x}, \mathbf{k})$ as

$$
W^{(0)}(t, \mathbf{x}, \mathbf{k})=\sum_{\tau, i, j} a_{i j}^{\tau}(t, \mathbf{x}, \mathbf{k}) B^{\tau, i j}(\mathbf{x}, \mathbf{k}),
$$

where $a_{i j}^{\tau}$ are scalar functions and $B^{\tau, i j}$ are $N \times N$ matrices defined in terms of the eigenvectors of the dispersion matrix of the system (see [22] for details). Under the 
appropriate diffusive scaling, $t \rightarrow \varepsilon^{2} t, \mathbf{x} \rightarrow \varepsilon \mathbf{x}$, we expect to obtain in the limit a diffusion equation for the coherence matrices.

6.1. Diffusive limit for acoustic waves. The acoustic wave equations for velocity and pressure, $\mathbf{u}$ and $p$, are

$$
\begin{aligned}
& \rho \frac{\partial \mathbf{u}}{\partial t}(t, \mathbf{x})+\nabla p(t, \mathbf{x})=0 \\
& \kappa \frac{\partial p}{\partial t}(t, \mathbf{x})+\operatorname{div} \mathbf{u}(t, \mathbf{x})=0
\end{aligned}
$$

with $t \geqslant 0, \mathbf{x} \in \mathbb{R}^{3}$. In the general case both the density $\rho(\mathbf{x})$ and the compressibility $\kappa(\mathbf{x})$ depend on $\mathbf{x}$. We will consider only the homogeneous background case, $\rho, \kappa=$ const. The sound speed is then constant, $v=\frac{1}{\sqrt{\kappa \rho}}$.

In the absence of polarization the radiative transport system simplifies, and it is enough to consider one scalar equation for the amplitude:

$$
\varepsilon^{2} \partial_{t} a^{\varepsilon}+\varepsilon v \hat{\mathbf{k}} \cdot \nabla_{\mathbf{x}} a^{\varepsilon}=\int_{\left|\mathbf{k}^{\prime}\right|=|\mathbf{k}|} \sigma\left(\mathbf{k}, \mathbf{k}^{\prime}\right) a^{\varepsilon}\left(\mathbf{k}^{\prime}\right) d \Omega\left(\hat{\mathbf{k}}^{\prime}\right)-\Sigma(|\mathbf{k}|) a^{\varepsilon}(\mathbf{k}) .
$$

Here $d \Omega$ is the unit sphere surface element, $\hat{\mathbf{k}}=\mathbf{k} /|\mathbf{k}|$ and the total scattering cross-section is

$$
\Sigma(|\mathbf{k}|)=\int_{\left|\mathbf{k}^{\prime}\right|=|\mathbf{k}|} \sigma\left(\mathbf{k}, \mathbf{k}^{\prime}\right) d \Omega\left(\hat{\mathbf{k}}^{\prime}\right) .
$$

In (6.4) and in the rest of this section, to simplify the notation we will often write $a(\mathbf{k})$ for a function depending on $(t, \mathbf{x}, \mathbf{k})$ if no confusion can arise. We are considering only rotationally invariant scattering so that the differential scattering cross-section $\sigma\left(\mathbf{k}, \mathbf{k}^{\prime}\right)$ is a nonnegative function of $|\mathbf{k}|$ and $\hat{\mathbf{k}} \cdot \hat{\mathbf{k}}^{\prime}$ only. We will write $\sigma(r, \mu), r=|\mathbf{k}|$ and $\mu=\hat{\mathbf{k}} \cdot \hat{\mathbf{k}}^{\prime}$ to denote this function.

This radiative transport equation for the amplitude $a^{\varepsilon}$ is of the form of (1.4) with collision operator

$$
C(a)(\mathbf{k})=\int_{\left|\mathbf{k}^{\prime}\right|=|\mathbf{k}|} \sigma\left(|\mathbf{k}|, \hat{\mathbf{k}} \cdot \hat{\mathbf{k}}^{\prime}\right) a\left(\mathbf{k}^{\prime}\right) d \Omega\left(\hat{\mathbf{k}}^{\prime}\right)-\Sigma(|\mathbf{k}|) a(\mathbf{k}) .
$$

In this case the kinetic variable is $\hat{\mathbf{k}}=\omega \in S^{2}$ and $r=|\mathbf{k}|$ acts as a parameter. The averaging is done on spheres

$$
w(t, \mathbf{x}, r)=\int_{|\mathbf{k}|=r} a(\mathbf{k}) d \Omega(\hat{\mathbf{k}})
$$

so the limit is an equation for a function of $(t, \mathbf{x}, r)$. The "mass" $w(t, \mathbf{x}, r)$ represents the average of the amplitudes $a^{\varepsilon}(t, \mathbf{x}, \mathbf{k}$ ) over all (unit) directions of wave vectors $\mathbf{k}$ with $|\mathbf{k}|=r$. Note that the integration is carried over the unit sphere and $d \Omega(\hat{\mathbf{k}})$ is the surface measure of the unit sphere.

6.2. Hypotheses for the model. Now we check that the hypotheses (h0)-(h5) and assumptions (a1) and (a2) are all satisfied for this model. Note that here we are considering a vector-valued kinetic variable $\mathbf{k}$. 
6.2.1. Hypotheses (h0)-(h3). Hypothesis (h0) is obvious. To show that (h1) holds take any function $a$ and integrate the collision term

$$
\begin{array}{rl}
\int_{|\mathbf{k}|=r} & C(a)(\mathbf{k}) d \Omega(\hat{\mathbf{k}}) \\
& =\iint_{|\mathbf{k}|=\left|\mathbf{k}^{\prime}\right|=r} \sigma\left(|\mathbf{k}|, \hat{\mathbf{k}} \cdot \hat{\mathbf{k}}^{\prime}\right) a\left(\mathbf{k}^{\prime}\right) d \Omega\left(\hat{\mathbf{k}}^{\prime}\right) d \Omega(\hat{\mathbf{k}})-\int_{|\mathbf{k}|=r} \Sigma(|\mathbf{k}|) a(\mathbf{k}) d \Omega(\hat{\mathbf{k}}) \\
& =\int_{\left|\mathbf{k}^{\prime}\right|=r} a\left(\mathbf{k}^{\prime}\right) \Sigma\left(\left|\mathbf{k}^{\prime}\right|\right) d \Omega\left(\hat{\mathbf{k}}^{\prime}\right)-\int_{|\mathbf{k}|=r} \Sigma(|\mathbf{k}|) a(\mathbf{k}) d \Omega(\hat{\mathbf{k}})=0 .
\end{array}
$$

This means that $w(r)=\int a(\mathbf{k}) d \mathbf{k}$ is the conserved quantity in (6.4).

The operator $C$ is negative in $L^{2}([22])$

$$
\begin{aligned}
& \int_{|\mathbf{k}|=r} C(a)(\mathbf{k}) a(\mathbf{k}) d \Omega(\hat{\mathbf{k}}) \\
& \quad=-\frac{1}{2} \iint_{|\mathbf{k}|=\left|\mathbf{k}^{\prime}\right|=r}\left(a(\mathbf{k})-a\left(\mathbf{k}^{\prime}\right)\right)^{2} \sigma\left(|\mathbf{k}|, \hat{\mathbf{k}} \cdot \hat{\mathbf{k}}^{\prime}\right) d \Omega\left(\hat{\mathbf{k}}^{\prime}\right) d \Omega(\hat{\mathbf{k}}) \leqslant 0 .
\end{aligned}
$$

Solutions of $C(a)(\mathbf{k})=0$ are of the form $a(\mathbf{k})=M_{a}(|\mathbf{k}|)$. These are precisely the Maxwellians, which can be parameterized in terms of their mass $w(r)$. Also, $C$ defines a contraction in $L^{1}$ : Given two functions $a$ and $\bar{a}$,

$$
\begin{aligned}
\int_{|\mathbf{k}|=r} & \int_{\left|\mathbf{k}^{\prime}\right|=|\mathbf{k}|} \sigma\left(|\mathbf{k}|, \hat{\mathbf{k}} \cdot \hat{\mathbf{k}}^{\prime}\right)\left(a\left(\mathbf{k}^{\prime}\right)-\bar{a}\left(\mathbf{k}^{\prime}\right)\right) d \Omega\left(\hat{\mathbf{k}}^{\prime}\right) \operatorname{sgn}(a(\mathbf{k})-\bar{a}(\mathbf{k})) d \Omega(\hat{\mathbf{k}}) \\
\leqslant & \int_{\left|\mathbf{k}^{\prime}\right|=r}\left|a\left(\mathbf{k}^{\prime}\right)-\bar{a}\left(\mathbf{k}^{\prime}\right)\right| \int_{|\mathbf{k}|=r} \sigma\left(\left|\mathbf{k}^{\prime}\right|, \hat{\mathbf{k}} \cdot \hat{\mathbf{k}}^{\prime}\right) d \Omega(\hat{\mathbf{k}}) d \Omega\left(\hat{\mathbf{k}}^{\prime}\right) \\
\quad & \int_{\left|\mathbf{k}^{\prime}\right|=r}\left|a\left(\mathbf{k}^{\prime}\right)-\bar{a}\left(\mathbf{k}^{\prime}\right)\right| \Sigma\left(\left|\mathbf{k}^{\prime}\right|\right) d \Omega\left(\hat{\mathbf{k}}^{\prime}\right),
\end{aligned}
$$

hence (h2) follows.

Finally we obtain (f1) from the following H-Theorem. For fixed $r>0$ multiplying (6.4) by $a^{\varepsilon}$ and integrating over the unit sphere of wave number directions with $|\mathbf{k}|=r$ we obtain

$$
\begin{aligned}
\partial_{t} \int_{|\mathbf{k}|=r}\left(a^{\varepsilon}\right)^{2} d \Omega(\hat{\mathbf{k}})+\frac{v}{\varepsilon} \operatorname{div}_{\mathbf{x}} \int_{|\mathbf{k}|=r} \hat{\mathbf{k}}\left(a^{\varepsilon}\right)^{2} d \Omega(\hat{\mathbf{k}}) \\
\quad+\frac{1}{\varepsilon^{2}} \iint_{|\mathbf{k}|=\left|\mathbf{k}^{\prime}\right|=r} \sigma\left(r, \hat{\mathbf{k}} \cdot \hat{\mathbf{k}}^{\prime}\right)\left(a^{\varepsilon}\left(\mathbf{k}^{\prime}\right)-a^{\varepsilon}(\mathbf{k})\right)^{2} d \Omega(\hat{\mathbf{k}}) d \Omega\left(\hat{\mathbf{k}}^{\prime}\right)=0 .
\end{aligned}
$$


From here we have that for any $T>0$

$$
\begin{gathered}
\int_{0}^{T} \iint_{|\mathbf{k}|=r}\left|a^{\varepsilon}(t, \mathbf{x}, \mathbf{k})-w^{\varepsilon}(t, \mathbf{x}, r)\right| d \Omega(\hat{\mathbf{k}}) d \mathbf{x} d t \\
\quad \leqslant \int_{0}^{T} \iint_{|\mathbf{k}|=\left|\mathbf{k}^{\prime}\right|=r}\left|a^{\varepsilon}(\mathbf{k})-a^{\varepsilon}\left(\mathbf{k}^{\prime}\right)\right| d \Omega(\hat{\mathbf{k}}) d \Omega\left(\hat{\mathbf{k}}^{\prime}\right) d \mathbf{x} d t \\
\leqslant K^{s t} \varepsilon^{2}\left\|a^{\varepsilon}\right\|_{L^{2}} .
\end{gathered}
$$

6.2.2. Assumptions (a1), (a2), (h4) and (h5). Now taking two functions $a$ and $\bar{a}$ in $L^{1}\left(S^{2}\right)$ we have

$$
\iint_{|\mathbf{k}|=r}|C(a)-C(\bar{a})| d \Omega(\hat{\mathbf{k}}) d \mathbf{x} \leqslant K^{s t} \iint|a-\bar{a}| d \Omega(\hat{\mathbf{k}}) d \mathbf{x},
$$

which implies (a1). Assumption (a2) is obvious. Since for a Maxwellian function $M_{a}$

$$
\int_{|\mathbf{k}|=r} \hat{\mathbf{k}} M_{a}(|\mathbf{k}|) d \Omega(\hat{\mathbf{k}})=0
$$

the structural hypothesis (h4) is automatically satisfied. In order to calculate the formal limit (see section 4) we need to invert the problem

$$
\left\langle C^{\prime}\left(\phi_{0}\right), \phi_{1}\right\rangle=v \hat{\mathbf{k}} \cdot \nabla_{\mathbf{x}} \phi_{0}
$$

for a Maxwellian $\phi_{0}$. In our case this equation is

$$
\int_{\left|\mathbf{k}^{\prime}\right|=|\mathbf{k}|} \sigma\left(|\mathbf{k}|, \hat{\mathbf{k}} \cdot \hat{\mathbf{k}}^{\prime}\right) \phi_{1}\left(\mathbf{k}^{\prime}\right) d \Omega\left(\hat{\mathbf{k}}^{\prime}\right)-\Sigma(|\mathbf{k}|) \phi_{1}(\mathbf{k})=v \hat{\mathbf{k}} \cdot \nabla_{\mathbf{x}} \phi_{0}
$$

where $\phi_{0}=\phi_{0}(t, \mathbf{x},|\mathbf{k}|)$.

Following [22, Section 5.1] the function $\hat{\mathbf{k}} \cdot \nabla_{\mathbf{x}} \phi_{0}(t, \mathbf{x},|\mathbf{k}|)$ is an eigenfunction of the operator $\mathbf{A}$ defined by

$$
\mathbf{A} f(\mathbf{k})=\int_{\left|\mathbf{k}^{\prime}\right|=|\mathbf{k}|} \sigma\left(|\mathbf{k}|, \hat{\mathbf{k}} \cdot \hat{\mathbf{k}}^{\prime}\right) f\left(\mathbf{k}^{\prime}\right) d \Omega\left(\hat{\mathbf{k}}^{\prime}\right)
$$

corresponding to the eigenvalue

$$
\lambda(|\mathbf{k}|)=2 \pi \int_{-1}^{1} \sigma(|\mathbf{k}|, \mu) \mu d \mu
$$


This can be seen as follows. Fix $\mathbf{k}=r \hat{\mathbf{k}}, \mathbf{a} \in \mathbb{R}^{3}$ and let $Q$ be a rotation such that $Q \hat{\mathbf{k}}=\mathbf{e}_{1}$, the first unit vector. Then, $\hat{\mathbf{k}} \cdot \mathbf{a}=\mathbf{e}_{1} \cdot Q \mathbf{a}$ and

$$
\begin{aligned}
\mathbf{A}(\mathbf{k} \mapsto \hat{\mathbf{k}} \cdot \mathbf{a})(\mathbf{k}) & =\int_{\left|\mathbf{k}^{\prime}\right|=r} \sigma\left(|\mathbf{k}|, \hat{\mathbf{k}} \cdot \hat{\mathbf{k}}^{\prime}\right)\left(\hat{\mathbf{k}}^{\prime} \cdot \mathbf{a}\right) d \Omega\left(\hat{\mathbf{k}}^{\prime}\right) \\
& =\int_{\left|\mathbf{k}^{\prime \prime}\right|=r} \sigma\left(r, Q \hat{\mathbf{k}} \cdot \hat{\mathbf{k}}^{\prime \prime}\right)\left(Q^{T} \hat{\mathbf{k}}^{\prime \prime} \cdot \mathbf{a}\right) d \Omega\left(\hat{\mathbf{k}}^{\prime \prime}\right) \\
& =\int_{\left|\mathbf{k}^{\prime}\right|=r} \sigma\left(r, \hat{\mathbf{k}}^{\prime} \cdot \mathbf{e}_{1}\right)\left(\hat{\mathbf{k}}^{\prime} \cdot \sum_{j}(Q \mathbf{a})_{j} \mathbf{e}_{j}\right) d \Omega\left(\hat{\mathbf{k}}^{\prime}\right) \\
& =\left(2 \pi \int_{-1}^{1} \sigma(r, \mu) \mu d \mu\right) \hat{\mathbf{k}} \cdot \mathbf{a},
\end{aligned}
$$

where we used $(Q \mathbf{a})_{1}=\hat{\mathbf{k}} \cdot \mathbf{a}$, the substitution $\hat{\mathbf{k}}^{\prime \prime}=Q \hat{\mathbf{k}}^{\prime}$ and the formula

$$
\int \sigma\left(r, \hat{k}_{i}^{\prime}\right) \hat{k}_{j}^{\prime} d \Omega\left(\hat{\mathbf{k}}^{\prime}\right)=\left(2 \pi \int_{-1}^{1} \sigma(r, \mu) \mu d \mu\right) \delta_{i j}
$$

(which is seen by expressing the integral in spherical coordinates).

A special solution of $(6.5)$ is

$$
\Phi_{1}(t, \mathbf{x}, \mathbf{k})=-\frac{v}{\Sigma(|\mathbf{k}|)-\lambda(|\mathbf{k}|)} \hat{\mathbf{k}} \cdot \nabla_{\mathbf{x}} \phi_{0}(t, \mathbf{x},|\mathbf{k}|) .
$$

The null space of $C^{\prime}\left(\phi_{0}\right)=C(\cdot)$ is the family of Maxwellians, thus the general solution of 6.5 is

$$
\phi_{1}(t, \mathbf{x}, \mathbf{k})=\Phi_{1}(t, \mathbf{x}, \mathbf{k})+\theta(t, \mathbf{x},|\mathbf{k}|) .
$$

From (4.3), the total mass

$$
w(t, \mathbf{x}, r)=\int_{|\mathbf{k}|=r} \phi_{0}(t, \mathbf{x},|\mathbf{k}|) d \Omega(\hat{\mathbf{k}})=4 \pi \phi_{0}(t, \mathbf{x}, r)
$$

satisfies the parabolic equation

$$
\begin{aligned}
\partial_{t} w(t, \mathbf{x}, r) & =\partial_{x_{i}} \partial_{x_{j}} \int_{|\mathbf{k}|=r} \hat{k}_{i} \hat{k}_{j} \frac{v^{2}}{\Sigma(|\mathbf{k}|)-\lambda(|\mathbf{k}|)} \phi_{0}(t, \mathbf{x},|\mathbf{k}|) d \Omega(\hat{\mathbf{k}}) \\
& =\frac{1}{3} \frac{v^{2}}{\Sigma(|\mathbf{k}|)-\lambda(|\mathbf{k}|)} \Delta_{x} w(t, \mathbf{x}, r) .
\end{aligned}
$$

6.3. Compactness. We will now complete the rigorous validation of the diffusive limit by establishing the compactness assumption.

Theorem 9. If the initial data satisfies

$$
\sup _{\varepsilon>0}\left\|a_{0}^{\varepsilon}\right\|_{L^{\infty}}+\left\|a_{0}^{\varepsilon}\right\|_{L^{1}}+\left\|D_{\mathbf{x}} a_{0}^{\varepsilon}\right\|_{L^{1}}<\infty
$$

then, for $r$ fixed, $\left\{w^{\varepsilon}(\cdot, \cdot, r)\right\}$ is sequentially precompact in $C\left([0, T], L_{\mathrm{loc}}^{1}\left(\mathbb{R}^{3}\right)\right)$ and any limit point $w$ solves, in the dissipative sense, the diffusion equation

$$
\partial_{t} w(t, \mathbf{x}, r)=\operatorname{div}_{\mathbf{x}}\left[D(r) \nabla_{\mathbf{x}} w(t, \mathbf{x}, r)\right]
$$


where the diffusion coefficient is as in 22$]$

$$
D(|\mathbf{k}|)=\frac{v^{2}}{3(\Sigma(|\mathbf{k}|)-\lambda(|\mathbf{k}|))} .
$$

The proof is based on the following lemma.

Lemma 10. For initial data satisfying (6.6), the set $\left\{w^{\varepsilon}(\cdot, \cdot, r)\right\}$ is, for each fixed $r$, sequentially precompact in $C\left([0, T], L_{\mathrm{loc}}^{1}\left(\mathbb{R}^{3}\right)\right)$.

Proof. This compactness is obtained by an argument analogous to Proposition 7. However, we need to invert the collision operator in order to obtain the same estimate. Consider the linear integral equation

$$
\begin{aligned}
g(\mathbf{k}) & =\Sigma(|\mathbf{k}|) f(\mathbf{k})-\int_{\left|\mathbf{k}^{\prime}\right|=|\mathbf{k}|} \sigma\left(|\mathbf{k}| \hat{\mathbf{k}} \cdot \hat{\mathbf{k}}^{\prime}\right) f\left(\mathbf{k}^{\prime}\right) d \Omega\left(\hat{\mathbf{k}}^{\prime}\right) \\
& =(\Sigma \mathbf{I}-\mathbf{A})(f)(\mathbf{k}),
\end{aligned}
$$

where $\mathbf{I}$ is the identity operator and $\mathbf{A}$ is a compact operator, both operators defined from $X:=L^{1}\left(S^{2}\right)$ to $X$ itself. The kernel of $\Sigma \mathbf{I}-\mathbf{A}$ is $N_{M}$, the set of Maxwellians, and has dimension 1. From the Fredholm theory for compact operators (see for example 18 for details) the range $\mathcal{R}(\Sigma \mathbf{I}-\mathbf{A})$ is closed and has codimension one. In fact it is also true that $N_{M}$ is the complementary space to the range, and we can invert the restriction of $\Sigma \mathbf{I}-\mathbf{A}$ on the quotient spaces:

$$
\begin{aligned}
\Sigma \mathbf{I}-\mathbf{A}: X / N_{M} & \rightarrow X / N_{M} \text { has an inverse } \\
\mathbf{K}: X / N_{M} & \rightarrow X / N_{M} .
\end{aligned}
$$

The inverse is a bounded linear operator:

$$
\|\mathbf{K} g\|_{L^{1}\left(S^{2}\right)} \leqslant\|K\|\|g\|_{L^{1}\left(S^{2}\right)}, \quad \text { for } g \in X / N_{M} .
$$

Now consider the equation (6.4). On the one hand we have

$$
\partial_{t} w^{\varepsilon}+\frac{v}{\varepsilon} \operatorname{div}_{\mathbf{x}} \int_{|\mathbf{k}|=r} \hat{\mathbf{k}} a^{\varepsilon} d \Omega(\hat{\mathbf{k}})=0 .
$$

On the other hand, upon inverting (6.4), we obtain

$$
\begin{aligned}
\frac{1}{\varepsilon} a^{\varepsilon} & =(\Sigma \mathbf{I}-\mathbf{A})^{-1}\left(-\varepsilon \partial_{t} a^{\varepsilon}-v \hat{\mathbf{k}} \cdot \nabla_{\mathbf{x}} a^{\varepsilon}\right) \\
& =-\varepsilon \partial_{t} \mathbf{K}\left(a^{\varepsilon}\right)-v \partial_{x_{i}} \mathbf{K}\left(\hat{k}_{i} a^{\varepsilon}\right),
\end{aligned}
$$

and therefore $w^{\varepsilon}$ satisfies the approximation equation

$$
\begin{gathered}
\partial_{t}\left(w^{\varepsilon}-\varepsilon v \partial_{x_{i}} H_{i}^{\varepsilon}\right)=v^{2} \partial_{x_{i}} \partial_{x_{j}} G_{i j}^{\varepsilon}, \\
\text { with } H_{i}^{\varepsilon}=\int_{|\mathbf{k}|=r} \hat{k}_{i} \mathbf{K}\left(a^{\varepsilon}\right) d \Omega(\hat{\mathbf{k}}) \text { and } G_{i j}^{\varepsilon}=\int_{|\mathbf{k}|=r} \hat{k}_{j} \mathbf{K}\left(\hat{k}_{i} a^{\varepsilon}\right) d \Omega(\hat{\mathbf{k}}) .
\end{gathered}
$$

The $L^{1}$-contraction property and the uniform BV bounds for the data (6.6) imply estimates for the $L^{1}$-moduli of continuity:

$$
\iint_{|\mathbf{k}|=r}\left|a^{\varepsilon}(t, \mathbf{x}+\mathbf{h}, \mathbf{k})-a^{\varepsilon}(t, \mathbf{x}+\mathbf{h}, \mathbf{k})\right| d \Omega(\hat{\mathbf{k}}) d \mathbf{x} \leqslant K^{s t}|\mathbf{h}|
$$


and, using (6.7),

$$
\begin{gathered}
\int\left|H_{i}^{\varepsilon}(t, \mathbf{x}+\mathbf{h}, r)-H_{i}^{\varepsilon}(t, \mathbf{x}, r)\right| d x \leqslant K^{s t}|\mathbf{h}| \\
M_{G_{i j}^{\varepsilon}}(\tau, \mathbf{h})=\int_{t}^{t+\tau} \sup _{\substack{|\mathbf{y}|<|\mathbf{h}|\\
}}\left|G_{i j}^{\varepsilon}(s, \mathbf{x}+\mathbf{y}, r)-G_{i j}^{\varepsilon}(s, \mathbf{x}, r)\right| d \mathbf{x} d s \\
\leqslant K^{s t} \int_{t}^{t+\tau} \iint_{|\mathbf{k}|=r}\left|a^{\varepsilon}(s, \mathbf{x}+\mathbf{h}, \mathbf{k})-a^{\varepsilon}(s, \mathbf{x}+\mathbf{h}, \mathbf{k})\right| d \Omega(\hat{\mathbf{k}}) d \mathbf{x} d s \\
\leqslant K^{s t} \tau|\mathbf{h}| .
\end{gathered}
$$

We then deduce the compactness of $\left\{w^{\varepsilon}\right\}$ by using Lemma 5 and an argument as in the proof of Proposition 7.

Proof of Theorem 9. Fix $r>0$. Along a subsequence,

$$
w^{\varepsilon}(t, \mathbf{x}, r)=\int_{|k|=r} a^{\varepsilon}(t, \mathbf{x}, \mathbf{k}) d \Omega(\hat{\mathbf{k}}) \rightarrow w(t, \mathbf{x}, r) \text { for a.e. }(t, \mathbf{x}) .
$$

From the H-estimate

$$
\begin{gathered}
\int_{0}^{T} \iint_{|\mathbf{k}|=r}\left|a^{\varepsilon}(t, \mathbf{x}, \mathbf{k})-w^{\varepsilon}(t, \mathbf{x}, r)\right| d \Omega(\hat{\mathbf{k}}) d \mathbf{x} d t \rightarrow 0 \\
a^{\varepsilon}(t, \mathbf{x}, \mathbf{k}) \rightarrow 4 \pi w(t, \mathbf{x}, r) \quad \text { for a.e. }(t, x) \text { and } d \Omega \text {-a.e. } \hat{\mathbf{k}} \text {. }
\end{gathered}
$$

At this point we have validated (f1) and (f2), and we can conclude by invoking Theorem 6. In any case we also give a formal direct argument. We can pass to the limit in (6.8) using (6.7) and the fact that $H_{i}^{\varepsilon}$ is uniformly bounded in $B V_{x}$ to conclude that $w$ satisfies

$$
\partial_{t} w=v^{2} \partial_{x_{i}} \partial_{x_{j}} 4 \pi \int_{|\mathbf{k}|=r} \hat{k}_{i} \mathbf{K}\left(w k_{j}\right) d \Omega(\hat{\mathbf{k}}) .
$$

Note that $g_{j}=\mathbf{K}\left(k_{j} w\right)$ if and only if $(\Sigma \mathbf{I}-\mathbf{A}) g_{j}=w k_{j}$, hence (6.2.2) implies $g_{j}=\frac{w}{\Sigma-\lambda} k_{j}$ and $w$ satisfies

$$
\partial_{t} w=\frac{v^{2}}{\Sigma(r)-\lambda(r)} \partial_{x_{i}} \partial_{x_{j}} 4 \pi w \int_{|\mathbf{k}|=r} \hat{k}_{i} k_{j} d \Omega(\hat{\mathbf{k}})=D(r) \Delta_{\mathbf{x}} w .
$$

\section{CompaCtNess OF MASS IN THE DIFFusive SCALING}

In this section we establish the compactness property $(\underline{\mathrm{f2}}$ ) in the diffusive scaling

$$
\begin{aligned}
& \partial_{t} f^{\varepsilon}+\frac{1}{\varepsilon} a(\xi) \cdot \nabla_{x} f^{\varepsilon}=\frac{1}{\varepsilon^{2}} C\left(f^{\varepsilon}\right), \\
& f^{\varepsilon}(0, x, \xi)=f_{0}^{\varepsilon}(x, \xi) .
\end{aligned}
$$

Apart from (h4) and (h5), we need some extra assumptions on the linearized collision operator (see below). (These are not too restrictive and are valid for the examples of section [5 and [6.) We also need a strengthened version of (f1),

$$
\int_{0}^{T} \iint\left|f^{\varepsilon}-\mathcal{M}\left(u^{\varepsilon}\right)\right|^{2} d \xi d x d t=O\left(\varepsilon^{2}\right) \quad \text { as } \varepsilon \rightarrow 0
$$

which in applications follows from an H-theorem. 
In the Taylor expansion (4.7),

$$
\begin{aligned}
& C\left(f_{0}+f_{1}\right)=C\left(f_{0}\right)+C^{\prime}\left(f_{0}\right) f_{1}+\bar{C}^{\prime \prime}\left(f_{0}\right):\left(f_{1}, f_{1}\right), \\
& \bar{C}^{\prime \prime}\left(f_{0}\right):\left(f_{1}, f_{1}\right):=\left\langle\int_{0}^{1} \int_{0}^{t} C^{\prime \prime}\left(f_{0}+s f_{1}\right) d s d t,\left(f_{1}, f_{1}\right)\right\rangle,
\end{aligned}
$$

we set $f_{0}=\mathcal{M}^{\varepsilon}:=\mathcal{M}\left(u^{\varepsilon}\right)$ and $f_{1}=f_{M}^{\varepsilon}:=f^{\varepsilon}-\mathcal{M}^{\varepsilon}$, and use it along with (h3 ) in (1.4) to obtain

$$
\frac{1}{\varepsilon} C^{\prime}\left(\mathcal{M}^{\varepsilon}\right) f_{M}^{\varepsilon}=\varepsilon \partial_{t} f^{\varepsilon}+a(\xi) \cdot \nabla_{x} f^{\varepsilon}-\frac{1}{\varepsilon} \bar{C}^{\prime \prime}\left(\mathcal{M}^{\varepsilon}\right):\left(f_{M}^{\varepsilon}, f_{M}^{\varepsilon}\right) .
$$

If we follow the compactness statements we proved in the previous sections we see that we want to invert the operator $\mathbf{C}_{u}:=C^{\prime}(\mathcal{M}(u))$ in order to estimate the second term of (1.5),

$$
\partial_{t} u^{\varepsilon}+\frac{1}{\varepsilon} \operatorname{div}_{x} \int a(\xi) f^{\varepsilon} d \xi=0
$$

which due to (h4) we can write as

$$
\partial_{t} u^{\varepsilon}+\frac{1}{\varepsilon} \operatorname{div}_{x} \int a(\xi)\left(f^{\varepsilon}-\mathcal{M}\left(u^{\varepsilon}\right)\right) d \xi=0 .
$$

This motivates the following assumptions for the collision operator. Let $X=$ $L^{1}(\mathbb{X}), N(u)$ be the null space of $\mathbf{C}_{u}$ and let $R(u)$ be its range. We will assume that

$$
\operatorname{dim} N(u)=\operatorname{codim} R(u)=1 \text { for every } u \in \mathbb{R} .
$$

Note that the first part of (h5) already implies $\operatorname{dim} N(u)=1$. Of course we can define the inverse of $\mathbf{C}_{u}$ on $R(u)$ with values in the quotient space $X / N(u)$ or equivalently in a complementary space to $N(u), R^{*}(u)$,

$$
\mathbf{D}_{u}:=\mathbf{C}_{u}^{-1}: R(u) \rightarrow R^{*}(u),
$$

which is bounded due to the fact that $R(u)$ is the range of a linear operator with finite codimension, and thus closed. It is convenient however to consider a pseudoinverse of $\mathbf{C}_{u}$, which is guaranteed to exist since this operator has finite index: There exist operators $\mathbf{T}_{u}, \mathbf{K}_{1, u}, \mathbf{K}_{2, u}: X \rightarrow X, \mathbf{T}_{u}$ bounded, $\mathbf{K}_{1, u}$ and $\mathbf{K}_{2, u}$ compact, such that

$$
\mathbf{T}_{u} \mathbf{C}_{u}=I+\mathbf{K}_{1, u} \text { and } \mathbf{C}_{u} \mathbf{T}_{u}=I+\mathbf{K}_{2, u},
$$

where $I$ is the identity. We will use this in particular in the following way. Given $g \in R^{*}(u)$ and $h$ in $X$ such that

$$
\mathbf{C}_{u}(g)=h,
$$

obviously $h \in R(u)$. Since $g$ is in the complementary space $R^{*}(u)$, we can invert this relation and get

$$
\begin{aligned}
g & =\mathbf{D}_{u} h=\mathbf{D}_{u} I h=\mathbf{D}_{u}\left(\mathbf{C}_{u} \mathbf{T}_{u}-\mathbf{K}_{2, u}\right) h \\
& =\mathbf{D}_{u} \mathbf{C}_{u} \mathbf{T}_{u} h-\mathbf{D}_{u} \mathbf{K}_{2, u} h \\
& =\mathbf{T}_{u} h-\mathbf{D}_{u} \mathbf{K}_{2, u} h .
\end{aligned}
$$

Note that since both $h$ and $\mathbf{C}_{u} \mathbf{T}_{u} h$ are in $R(u)$, then so is $\mathbf{K}_{2, u} h$, so that the last expression is well defined. Note also that the operator $\mathbf{D}_{u} \mathbf{K}_{2, u}$ is compact, defined on the whole space $X$ and thus

$$
g=\mathbf{S}_{u} h,
$$


where $\mathbf{S}_{u}: X \rightarrow X$ is a bounded operator. We further assume that this operator depends smoothly on the parameter $u$ and is uniformly bounded:

$$
\left\|\mathbf{S}_{u} g\right\|_{X} \leqslant \kappa_{1}\|g\|_{X}
$$

for some constant $\kappa_{1}$ independent of $u$, and

$$
\left\{\begin{array}{l}
\text { The mapping } \quad \mathbb{R} \ni u \mapsto \mathbf{S}_{u} \in \mathcal{L}(X, X) \\
\text { is } C^{2} \text { with respect to the norm topology, and its } \\
\text { derivatives are bounded uniformly in } u
\end{array}\right.
$$

We will denote the first derivative of $\mathbf{S}_{u}$ by $\mathbf{L}_{u}$ and the second by $\mathbf{J}_{u}$.

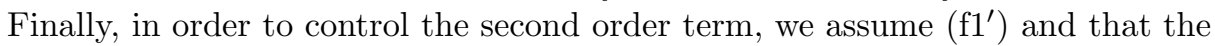
collision operator is twice differentiable and satisfies

$$
\left\|\bar{C}^{\prime \prime}(h):(g, g)\right\|_{X} \leqslant \kappa_{2}\|g\|_{L^{2}(\mathbb{X})}^{2}, \quad \text { for every } h \in L^{\infty} \cap L^{1}(\mathbb{X}),
$$

where $\kappa_{2}$ is a constant independent of $h$.

With these assumptions we can state the following compactness result.

Proposition 11. With the above assumptions on the collision operator, (h0)-(h9), assumption (f1'), and for $|a(\xi)| \leqslant M$, if the initial data satisfies

$$
\sup _{\varepsilon>0}\left\|f_{0}^{\varepsilon}\right\|_{L^{\infty}}+\left\|f_{0}^{\varepsilon}\right\|_{L^{1}}+\left\|D_{x} f_{0}^{\varepsilon}\right\|_{L^{1}}<\infty
$$

then the set $\left\{u^{\varepsilon}\right\}$, where $u^{\varepsilon}(t, x)=\int f^{\varepsilon}(t, x, \xi) d \xi$, is sequentially precompact in $C\left([0, T], L_{\mathrm{loc}}^{1}\left(\mathbb{R}^{d}\right)\right)$ for any $T>0$.

Proof. 1. From (1.4) we obtained (7.1). Using (7.3) here we can write

$$
\frac{1}{\varepsilon} f_{M}^{\varepsilon}=\varepsilon \mathbf{S}_{u} \partial_{t} f^{\varepsilon}+\mathbf{S}_{u} a \cdot \nabla_{x} f^{\varepsilon}-\mathbf{S}_{u} g^{\varepsilon},
$$

where

$$
g^{\varepsilon}:=\frac{1}{\varepsilon} \bar{C}^{\prime \prime}:\left(f_{M}^{\varepsilon}, f_{M}^{\varepsilon}\right)
$$

We want to bring the derivatives in $t$ and $x$ out of the operator $\mathbf{S}_{u}$, but since now this depends on $u^{\varepsilon}(t, x)$ we need to account for the derivative of the operator. Thus we have

$$
\begin{aligned}
\varepsilon^{-1} f_{M}^{\varepsilon}= & \varepsilon \partial_{t} \mathbf{S}_{u}\left(f^{\varepsilon}\right)+\sum_{j} \partial_{x_{j}} \mathbf{S}_{u}\left(a_{j} f^{\varepsilon}\right)-\mathbf{S}_{u} g^{\varepsilon} \\
& -\mathbf{L}_{u}\left(\varepsilon u_{t}^{\varepsilon} f^{\varepsilon}\right)-\mathbf{L}_{u}\left(f^{\varepsilon} a \cdot \nabla_{x} u^{\varepsilon}\right) .
\end{aligned}
$$

Since $\varepsilon u_{t}^{\varepsilon}=-\operatorname{div}_{x} \int a(\xi) f^{\varepsilon} d \xi$ we can rewrite the last identity in the form

$$
\begin{aligned}
\varepsilon^{-1} f_{M}^{\varepsilon}= & \varepsilon \partial_{t} \mathbf{S}_{u}\left(f^{\varepsilon}\right)+\sum_{j} \partial_{x_{j}} \mathbf{S}_{u}\left(a_{j} f^{\varepsilon}\right)-\mathbf{S}_{u} g^{\varepsilon} \\
& +\sum_{j} \partial_{x_{j}} \mathbf{L}_{u}\left(f^{\varepsilon} \int_{\xi^{\prime}} a_{j}\left(\xi^{\prime}\right) f^{\varepsilon}\left(\xi^{\prime}\right) d \xi^{\prime}-a_{j} f^{\varepsilon} \int_{\xi^{\prime}} f^{\varepsilon}\left(\xi^{\prime}\right) d \xi^{\prime}\right) .
\end{aligned}
$$

We now multiply this by $a_{i}(\xi)$ and integrate in $\xi$, substituting in (7.2) to obtain

$$
\begin{aligned}
\partial_{t} u^{\varepsilon} & =-\frac{1}{\varepsilon} \sum_{i} \partial_{x_{i}} \int a_{i}\left(f^{\varepsilon}-\mathcal{M}\left(u^{\varepsilon}\right)\right) d \xi \\
& =\varepsilon \partial_{t} \operatorname{div}_{x} A_{\varepsilon}-\operatorname{div}_{x} B_{\varepsilon}+\sum_{i, j} \partial_{x_{i}} \partial_{x_{j}}\left(C_{\varepsilon}^{i j}+D_{\varepsilon}^{i j}\right),
\end{aligned}
$$


where

$$
\begin{aligned}
& A_{\varepsilon}=\int a(\xi) \mathbf{S}_{u} f^{\varepsilon} d \xi, \quad B_{\varepsilon}=\int a(\xi) \mathbf{S}_{u} g^{\varepsilon} d \xi, \quad C_{\varepsilon}^{i j}=\int a_{i} \mathbf{S}_{u}\left(a_{j} f^{\varepsilon}\right) d \xi \\
& \text { and } D_{\varepsilon}^{i j}=\int a_{i} \mathbf{L}_{u}\left(f^{\varepsilon} \int_{\xi^{\prime}}\left(a_{j}\left(\xi^{\prime}\right)-a_{j}(\xi)\right) f^{\varepsilon}\left(\xi^{\prime}\right) d \xi^{\prime}\right) d \xi
\end{aligned}
$$

2. The idea now is the same as in the proof of Lemma 10. From the contraction property and the BV bounds on the initial data we get an $L^{1}$-modulus of continuity for $u^{\varepsilon}$ in $x$. To estimate the $t$-modulus of continuity we need to estimate the $x$ modulus of continuity of the terms on the right-hand side of (7.4). This now requires more technical computations since the operators involved in these terms depend on $x$ through $u^{\varepsilon}$.

3. Estimate for $A_{\varepsilon}$

$$
\begin{aligned}
\int \mid A_{\varepsilon}(x+h)- & A_{\varepsilon}(x) \mid d x \\
= & \int\left|\int a(\xi)\left(\mathbf{S}_{u(x+h)} f^{\varepsilon}(x+h)-\mathbf{S}_{u(x)} f^{\varepsilon}(x)\right) d \xi\right| d x \\
\leqslant & \int\left|\int a(\xi)\left(\mathbf{S}_{u(x+h)}-\mathbf{S}_{u(x)}\right) f^{\varepsilon}(x+h) d \xi\right| d x \\
& +\int\left|\int a(\xi) \mathbf{S}_{u(x)}\left(f^{\varepsilon}(x+h)-f^{\varepsilon}(x)\right) d \xi\right| d x \\
= & : A^{1}+A^{2}
\end{aligned}
$$

Since we can write

$$
\left(\mathbf{S}_{u(x+h)}-\mathbf{S}_{u(x)}\right) f=\int_{0}^{1}\left(u^{\varepsilon}(x+h)-u^{\varepsilon}(x)\right) \mathbf{L}_{v(s)} f d s,
$$

where $v(s):=s u^{\varepsilon}(x+h)+(1-s) u^{\varepsilon}(x)$, for the first of the above terms we have

$$
\begin{aligned}
A^{1} & \leqslant M \int\left|u^{\varepsilon}(x+h)-u^{\varepsilon}(x)\right| \int_{0}^{1}\left|\int \mathbf{L}_{v(s)} f^{\varepsilon}(x+h) d \xi\right| d s d x \\
& \leqslant K^{s t} \int\left|u^{\varepsilon}(x+h)-u^{\varepsilon}(x)\right| d x\left\|u^{\varepsilon}(\cdot+h)\right\|_{L^{\infty}}=O(|h|) .
\end{aligned}
$$

Here we have used the a priori $L^{\infty}$ bounds, (h8) and the $x$-modulus of continuity of $u^{\varepsilon}$. For the second term, $A^{2}$, similarly we have

$$
\begin{aligned}
A^{2} & =\int\left|\int a(\xi) \mathbf{S}_{u}\left(f^{\varepsilon}(x+h)-f^{\varepsilon}(x)\right) d \xi\right| d x \\
& \leqslant K^{s t} \iint\left|f^{\varepsilon}(x+h)-f^{\varepsilon}(x)\right| d \xi d x=O(|h|),
\end{aligned}
$$

hence we also have

$$
\int\left|A_{\varepsilon}(x+h)-A_{\varepsilon}(x)\right| d x=O(|h|) .
$$


4. Estimates for $C_{\varepsilon}^{i j}$ and $D_{\varepsilon}^{i j}$. The terms $C_{\varepsilon}^{i j}$ can be estimated exactly in the same way as above to obtain

$$
\int\left|C_{\varepsilon}^{i j}(x+h)-C_{\varepsilon}^{i j}(x)\right| d x=O(|h|) .
$$

The same idea applies to the terms $D_{\varepsilon}^{i j}$, but for these we need to observe that the bound on the integrand is obtained in a slightly different way. More specifically we have

$$
\begin{aligned}
& \int\left|D_{\varepsilon}^{i j}(x+h)-D_{\varepsilon}^{i j}(x)\right| d x \\
& \leqslant \int\left|u^{\varepsilon}(x+h)-u^{\varepsilon}(x)\right| \int_{0}^{1}\left|\int a(\xi) \mathbf{J}_{v(s)} F^{\varepsilon}(x+h) d \xi\right| d s d x \\
& \quad+\int\left|\int a(\xi) \mathbf{L}_{u(x)}\left(F^{\varepsilon}(x+h)-F^{\varepsilon}(x)\right) d \xi\right| d x,
\end{aligned}
$$

where

$$
F^{\varepsilon}=f^{\varepsilon} \int\left(a\left(\xi^{\prime}\right)-a(\xi)\right) f^{\varepsilon}\left(\xi^{\prime}\right) d \xi^{\prime}
$$

Since

$$
\int\left|F^{\varepsilon}(t, x, \xi)\right| d \xi \leqslant K^{s t}\left\|f^{\varepsilon}(t, x, \cdot)\right\|_{L^{1}(\mathbb{X})}^{2} \leqslant K^{s t},
$$

using again (h8) we have the same type of estimate for the first of the above integrals:

$$
\int\left|u^{\varepsilon}(x+h)-u^{\varepsilon}(x)\right| \int_{0}^{1}\left|\int a(\xi) \mathbf{J}_{v(s)} F^{\varepsilon}(x+h) d \xi\right| d s d x \leqslant K^{s t}|h| .
$$

To estimate the second integral note that

$$
\begin{aligned}
\left|F^{\varepsilon}(x+h)-F^{\varepsilon}(x)\right| \leqslant & K^{s t}\left|f^{\varepsilon}(x+h)-f^{\varepsilon}(x)\right|\left\|f^{\varepsilon}\right\|_{L^{1}(\mathbb{X})} \\
& +K^{s t}\left|f^{\varepsilon}\right|\left\|f^{\varepsilon}(x+h)-f^{\varepsilon}(x)\right\|_{L^{1}(\mathbb{X})} .
\end{aligned}
$$

Hence again from (h8) we finally get

$$
\int\left|D_{\varepsilon}^{i j}(x+h)-D_{\varepsilon}^{i j}(x)\right| d x=O(|h|) .
$$

5. Estimate for $B_{\varepsilon}$. For the term $B_{\varepsilon}$, as there is no information on its modulus of continuity, we proceed to show it gives rise to an error term. From (h7), (h9) and $\left(\underline{f 1^{\prime}}\right)$ we obtain

$$
\begin{aligned}
\int_{0}^{t} \int \mid & B_{\varepsilon}(t, x)\left|d x d t=\int_{0}^{t} \int\right| \int S_{u} g^{\varepsilon} d \xi\left|d x d t \leqslant K^{s t} \int_{0}^{t} \iint\right| g^{\varepsilon} \mid d \xi d x d t \\
& =\int_{0}^{t} \iint\left|\frac{1}{\varepsilon} \bar{C}^{\prime \prime}:\left(f_{M}^{\varepsilon}, f_{M}^{\varepsilon}\right)\right| d \xi d x d t \leqslant K^{s t} \frac{1}{\varepsilon} \int_{0}^{t} \iint\left|f^{\varepsilon}-\mathcal{M}^{\varepsilon}\right|^{2} d \xi d x d t \\
& =O(\varepsilon) .
\end{aligned}
$$

6. Proceeding as in the proof of Proposition 7 , we use (7.5), (7.8), (7.6) and (7.7) to obtain

$$
\int\left|u^{\varepsilon}(t+\tau, x)-u^{\varepsilon}(t, x)\right| d x \leqslant K^{s t}\left(\delta+\varepsilon+\varepsilon \frac{1}{\delta}+\tau h\left(\frac{1}{\delta^{2}}+\frac{1}{h^{2}}\right)\right),
$$


where the last three terms are the respective contributions of the last three terms in the right hand side of (7.4). Optimizing in $\delta$ and $h$ we again obtain

$$
\int\left|u^{\varepsilon}(t+\tau, x)-u^{\varepsilon}(t, x)\right| d x \leqslant K^{s t}\left(\varepsilon\left(1+\frac{1}{\tau^{1 / 2}}\right)+\tau^{1 / 2}\right) .
$$

7. Recall now that

$$
\int\left|u^{\varepsilon}(s, x+h)-u^{\varepsilon}(s, x)\right| d x \leqslant K^{s t} \omega(h) .
$$

For each fixed $s$ we choose a subsequence $\left\{u^{\varepsilon_{n}}\right\}$ such that $u^{\varepsilon_{n}}(s, \cdot) \rightarrow u(s, \cdot)$ in $L^{1}(V)$, where $V$ is a compact subset of $\mathbb{R}^{d}$. By a diagonal argument we can extract a subsequence (still denoted by $\left\{u^{\varepsilon_{n}}\right\}$ ) for which this holds for every $s \in \mathbb{Q}$.

We want to show that $\left\{u^{\varepsilon_{n}}\right\}$ is a Cauchy sequence in $C\left([0, T] ; L^{1}(V)\right)$. Given $\delta>0$, choose $\tau<\delta^{2}$ and let $s_{1}<\ldots<s_{l}$ be rationals in $[0, T]$ such that $s_{1}$, $s_{i+1}-s_{i}$ and $T-s_{l}$ are all less than $\tau / 2$. Then, for any $t \in[0, T]$ we can find a $j$ such that $\left|t-s_{j}\right|<\tau$, and hence for any $n$ and $m$, using (7.9) we have

$$
\begin{aligned}
& \int_{V}\left|u^{\varepsilon_{n}}(t, x)-u^{\varepsilon_{m}}(t, x)\right| d x \\
& \leqslant \int_{V}\left|u^{\varepsilon_{n}}(t, x)-u^{\varepsilon_{n}}\left(s_{j}, x\right)\right| d x+\int_{V}\left|u^{\varepsilon_{n}}\left(s_{j}, x\right)-u^{\varepsilon_{m}}\left(s_{j}, x\right)\right| d x \\
& \quad+\int_{V}\left|u^{\varepsilon_{m}}\left(s_{j}, x\right)-u^{\varepsilon_{m}}(t, x)\right| d x \\
& \leqslant\left(\frac{\varepsilon_{n}+\varepsilon_{m}}{\tau^{1 / 2}}+2 \delta\right)+\sup _{1 \leqslant i \leqslant l} \int_{V}\left|u^{\varepsilon_{n}}\left(s_{i}, x\right)-u^{\varepsilon_{m}}\left(s_{i}, x\right)\right| d x .
\end{aligned}
$$

We can now choose $n$ and $m$ large enough to make this quantity less than, say, $4 \delta$.

Remark. It is clear that even though we have compactness in $L^{1}$, it is not possible to obtain the limiting equation with traditional techniques without further estimates. This is certainly a strength of using dissipative solutions. It should be pointed though that, for degenerate parabolic equations, it is not clear whether the framework of dissipative solutions provides uniqueness, as is the case with the entropy solutions of Chen-Perthame [8]. The latter, however, require much stronger regularity assumptions that are avoided here. For strictly parabolic equations the notions of strong and dissipative solutions are equivalent.

\section{ACKNOWLEDGMENT}

The first author was partially supported by the European Union IHP-project MCWAVE and NSF Grant 0074037. The second author was partially supported by the National Science Foundation, the European Union IHP-project HYKE, and the "Pythagoras" contract from the Greek Secretariat for Research. This research was completed at the Institute for Applied and Computational Mathematics, FORTH, Greece.

\section{REFERENCES}

[1] C. Bardos, F. Golse and B. Perthame, The Rosseland approximation for the radiative transfer equations, Comm. Pure Appl. Math. 40 (1987), no. 6, 691-721. MR0910950 (88j:35134) 
[2] C. Bardos, F. Golse and B. Perthame, Erratum to the article: "The Rosseland approximation for the radiative transfer equations" [Comm. Pure Appl. Math. 40 (1987), no. 6, 691-721], Comm. Pure Appl. Math. 42 (1989), no. 6, 891-894. MR.1003439 (90e:35133)

[3] C. Bardos, F. Golse, B. Perthame and R. Sentis, The nonaccretive radiative transfer equations: existence of solutions and Rosseland approximation, J. Funct. Anal. 77 (1988), no. 2, 434-460. MR0933978 (89f:35174)

[4] F. Bouchut, Construction of BGK models with a family of kinetic entropies for a given system of conservation laws, J. Statist. Phys. 95 (1999), 113-170. MR.1705583 (2000f:82084)

[5] F. Bouchut, F.R. Guarguaglini and R. Natalini, Diffusive BGK approximations for nonlinear multidimensional parabolic equations, Indiana Univ. Math. J. 49 (2000), 723-749. MR1793689 (2001k:35162)

[6] H. Brezis, Analyse fonctionnelle, Masson, Paris, 1983. MR0697382 (85a:46001)

[7] S. Chandrasekhar, Radiative Transfer, Dover, New York, 1960. MR.0111583(22:2446)

[8] G.-Q. Chen and B. Perthame, Well-posedness for non-isotropic degenerate parabolichyperbolic equations, Ann. Inst. H. Poicaré, Analyse Non-linéaire 20 (2003), no. 4, 645-668. MR.1981403 (2004c:35235)

[9] L. C. Evans, The perturbed test function method for viscosity solutions of nonlinear PDE, Proc. Roy. Soc. Edinburgh Sect. A 111 (1989), no. 3-4, 359-375. MR1007533 (91c:35017)

[10] L. Gosse and G. Toscani, Space localization and well-balanced schemes for discrete kinetic models in diffusive regimes, SIAM J. Numer. Anal. 41 (2003), no. 2, 641-658 (electronic). MR2004192 (2004g:65107)

[11] S. Hwang and A. E. Tzavaras, Kinetic decomposition of approximate solutions to scalar conservation laws: applications to relaxation and diffusion-dispersion approximations, Comm. Partial Differential Equations 27 (2002), pp. 1229-1254. MR1916562 (2004f:35115)

[12] S. Jin, L. Pareschi and G. Toscani, Uniformly accurate diffusive relaxation schemes for multiscale transport equations, SIAM J. Numer. Anal. 38 (2000), no. 3, 913-936 (electronic). MR:1781209 (2001h:65095)

[13] M. Katsoulakis and A.E. Tzavaras, Contractive relaxation systems and the scalar multidimensional conservation law, Communications in Partial Differential Equations 22 (1997), pp. 195-233. MR.1434144 (97m:35168)

[14] M. Katsoulakis and A.E. Tzavaras, Multiscale analysis for interacting particles: relaxation systems and scalar conservation laws, J. Statist. Phys. 96 (1999), 715-763. MR:1716813 (2000j:82029)

[15] S.N. Kruzhkov, First order quasilinear equations with several independent variables, Math. USSR Sbornik 10 (1970), pp. 217-243.

[16] T. G. Kurtz, Convergence of sequences of semigroups of nonlinear operators with an application to gas kinetics, Trans. Amer. Math. Soc. 186 (1973), 473-513. MR 0336482 (49:1256)

[17] C. Lattanzio and R. Natalini, Convergence of diffusive BGK approximations for nonlinear strongly parabolic systems, Proc. Royal Soc. Edinburgh (to appear)

[18] P. D. Lax, Functional analysis, Wiley-Intersci., New York, 2002. MR1892228 (2003a:47001)

[19] P. L. Lions and G. Toscani, Diffusive limits for finite velocity Boltzmann kinetic models, Revista Mat. Iberoamericana 13 (1997), pp. 256-272. MR1617393 (99g:76127)

[20] P. Marcati and B. Rubino, Hyperbolic to parabolic relaxation theory for quasilinear first order systems, J. Differential Equations 162 (2000), 359-399. MR1751710 (2001d:35125)

[21] R. Natalini, A discrete kinetic approximation of entropy solutions to multidimensional scalar conservation laws, J. Differential Equations 148 (1998), 292-317. MR1643175 (99e:35139)

[22] G. Papanicolaou and L. Ryzhik, Hyperbolic equations and frequency interactions (Park City, UT, 1995), 305-382, Amer. Math. Soc., Providence, RI. MR1662832 (99k:73063)

[23] B. Perthame and P.E. Souganidis, Accretive and entropy solutions to non-isotropic degenerate parabolic balance laws, Arch. Ration. Mech. Anal. 170 (2003), no. 4, 359-370. MR2022136 (2006c:35162a)

[24] B. Perthame and E. Tadmor, A kinetic equation with kinetic entropy functions for scalar conservation laws, Comm. Math. Phys. 136 (1991), pp. 501-517. MR.1099693 (92d:82095)

[25] M. Portilheiro, Weak solutions for equations defined by accretive operators I, Proc. Roy. Soc. Edinburgh Sect. A 133 (2003), no. 5, 1193-1207. MR2018332 (2004j:47130)

[26] M. Portilheiro, Weak solutions for equations defined by accretive operators II: relaxation limits, J. Differential Equations 195 (2003), no. 1, 66-81. MR2019243(2004j:47129) 
[27] A.E. Tzavaras, Derivation of fluid equations for kinetic models with one conserved quantity, In "Proceedings of International Conf. on Mathematical Analysis", National Techn. Univ. of Athens, Greece, 2003.

[28] A.E. Tzavaras, On the mathematical theory of fluid dynamic limits to conservation laws, Advances in Mathematical Fluid Mechanics (Paseky, 1999), pp. 192-222, Springer, Berlin, 2000. MR:1863213 (2003g:76003)

Institute of Applied and Computational Mathematics, FORTh, 71110 Heraklion, Crete, Greece

E-mail address: portilhe@tem.uoc.gr

Current address: Departamento de Matemática, Faculdade de Ciências e Tecnologia da Universidade de Coimbra, Apartado 3008, 3001-454 Coimbra, Portugal

E-mail address: portilhe@mat.uc.pt

Department of Mathematics, University of Wisconsin, Madison, Wisconsin 53706 and - Institute of Applied and Computational Mathematics, FORTH, 71110 Heraklion, Crete, Greece

E-mail address: tzavaras@math.wisc.edu

Current address: Department of Mathematics, University of Maryland, College Park, Maryland 20742

E-mail address: tzavaras@math.umd.edu 